\title{
PROJECTABLE VERONESE VARIETIES
}

\author{
ALBERTO ALZATI AND EDOARDO BALLICO
}

\begin{abstract}
Let $X$ be a non degenerate, reduced, reducible algebraic variety embedded in $\mathbb{P}^{N}$, of pure dimension $m \geq 3$. $X$ is said to be an $x$-projectable Veronese variety if, assuming $N \geq m+x+1, X$ is of minimal degree, connected in codimension 1 and isomorphically projectable into a linear space of dimension $m+x$.

In this paper we classify 2 and 3-projectable Veronese varieties and $x$ projectable Veronese varieties having only linear components.
\end{abstract}

\section{INTRODUCTION}

Let $\mathbb{P}^{N}$ be the $N$-dimensional projective space over $\mathbb{C}$. In this paper a variety will always be an algebraic, reduced, of pure dimension, projective scheme embedded in some projective space $\mathbb{P}^{N}$. For any integer $k \geq 0$, a variety $V \subset \mathbb{P}^{N}$ is said to be connected in codimension $k$ if for any subvariety $W \subset V$, such that $\operatorname{cod}_{V}(W)>k$, the algebraic set $V \backslash W$ is connected. For any variety $V \subset \mathbb{P}^{N}$ and for any $\lambda$ dimensional linear subspace $\Lambda \subset \mathbb{P}^{N}$ we say that $V$ projects isomorphically to $\Lambda$ if there exists a linear projection $\pi_{\mathcal{L}}: \mathbb{P}^{N}--->\Lambda$, from a suitable $(N-\lambda-1)$ dimensional linear space $\mathcal{L}$, disjoint from $V$, such that $\pi_{\mathcal{L}}(V)$ is isomorphic to $V$ via the projection $\pi_{\mathcal{L}}$.

In $[\mathrm{A}-\mathrm{B}]$ we have classified all reducible Veronese surfaces, according to the following definition.

Definition 1. For any positive integer $n \geq 1$, we will call reducible Veronese surface any algebraic surface $X \subset \mathbb{P}^{n+4}$ such that:

i) $X$ is a non degenerate, reducible variety of pure dimension 2 ;

ii) $\operatorname{deg}(X)=n+3, \operatorname{cod}(X)=n+2$, so that $X$ is a minimal degree surface;

iii) $\operatorname{dim}[\operatorname{Sec}(X)] \leq 4$, so that it is possible to choose a generic linear space $\mathcal{L}$ of dimension $n-1$ in $\mathbb{P}^{n+4}$ such $\pi_{\mathcal{L}}(X)$ is isomorphic to $X$ via $\pi_{\mathcal{L}}$, where $\pi_{\mathcal{L}}$ is the the rational projection $\pi_{\mathcal{L}}: \mathbb{P}^{n+4}--->\Lambda$, from $\mathcal{L}$ to a generic target $\Lambda \simeq \mathbb{P}^{4}$;

$i v) X$ is connected in codimension 1 , i.e. if we drop any finite number (eventually $0)$ of points $Q_{1}, \ldots, Q_{r}$ from $X$ we have $X \backslash\left\{Q_{1}, \ldots, Q_{r}\right\}$ is connected;

v) $X$ is a locally Cohen-Macaulay surface.

Examples of reducible Veronese surfaces are the surfaces $\Sigma_{n}$ introduced by the following:

Date: March, 30th 2010.

1991 Mathematics Subject Classification. Primary 14J25; Secondary 14N20.

Key words and phrases. reducible varieties, projectability.

This work is within the framework of the national research projects: "Geometry on Algebraic Varieties" Cofin 2008 of MIUR and "Geometric Properties of Real and Complex Varieties" Cofin 2007 of MIUR. 
Definition 2. For any positive integer $n \geq 1$, let us choose a plane $\Pi_{0}$ and $n+2$ distinct points $P_{1}, \ldots, P_{n+2}$ in general position in $\mathbb{P}^{n+4}$, so that $\left\langle\Pi_{0} \cup P_{1} \cup \ldots \cup P_{n+2}\right\rangle$ $=\mathbb{P}^{n+4}$. Let us choose $n+2$ planes $\Pi_{i}, i=1, \ldots, n+2, P_{i} \in \Pi_{i}$, such that $\Pi_{i} \cap \Pi_{0}$ is a line $L_{i}$ and the $n+2$ lines $L_{i}$ are in general position on $\Pi_{0}$. (i.e. the curve given by their union has no triple points). Let us call $\Sigma_{n}$ any surface in $\mathbb{P}^{n+4}$ which is the union $\Pi_{0} \cup \Pi_{1} \ldots \cup \Pi_{n+2}$.

In $[\mathrm{A}-\mathrm{B}]$ we proved the following:

Theorem 1. (see Theorems 2 and 3 of $[\mathrm{A}-\mathrm{B}]$ ) Let $X$ be a reducible Veronese surface. Then we have only three possibilities:

i) $X$ is a surface $\Sigma_{n}$ for some $n \geq 1$;

ii) $X=Q \cup X_{1} \cup X_{2}$, where $Q$ is a smooth quadric, $X_{1}$ and $X_{2}$ are planes, and $Q, X_{1}, X_{2}$ intersect transversally along a unique line $L:=Q \cap X_{1} \cap X_{2}$;

iii) $X=Q \cup X_{1} \cup X_{2}$ as above and $X_{1}$ and $X_{2}$ cut $Q$ along two lines intersecting at a point $P:=X_{1} \cap X_{2}$.

Here we will consider $x$-projectable Veronese varieties $X \subset \mathbb{P}^{N}$ of dimension $m \geq 3$, according to the following definition. Note that the $x$-projectability is a natural geometric property which a minimal degree algebraic subset may have.

Definition 3. For any positive integers $m \geq x \geq 2$, we will call x-projectable Veronese variety any algebraic variety $X \subset \mathbb{P}^{N}, N \geq m+x+1$, such that:

i) $X$ is a non degenerate, reducible variety of dimension $m \geq 3$;

ii) $\operatorname{deg}(X)=\operatorname{cod}(X)+1$, so that $X$ is a minimal degree variety;

iii) it is possible to choose a generic linear space $\mathcal{L}$ of dimension $N-m-1-x$ in $\mathbb{P}^{N}$ such $\pi_{\mathcal{L}}(X)$ is isomorphic to $X$ via $\pi_{\mathcal{L}}$, where $\pi_{\mathcal{L}}$ is the the rational projection $\pi_{\mathcal{L}}: \mathbb{P}^{N}--->\Lambda$, from $\mathcal{L}$ to a generic target $\Lambda \simeq \mathbb{P}^{m+x}$

$i v) X$ is connected in codimension $1, i$. e. it is possible to arrange the components of $X$ in such a way that $X=X_{1} \cup X_{2} \cup \ldots \cup X_{r}$ and $\operatorname{cod}_{X_{j}}\left[\left(X_{1} \cup \ldots \cup X_{j-1}\right) \cap X_{j}\right]=1$ for any $j \geq 2$;

v) $X$ is a locally Cohen-Macaulay variety.

Remark 1. Actually v) implies iv) by Corollary 2.4 of [Ha], however it is more useful to give the above definitions 1 and 3 because condition iv) is crucial to get the classifications. The assumption $m \geq x$ implies that $m+x \leq 2 m$.

Remark 2. Note that if condition iii) of Definition 3 is satisfied then $\operatorname{dim}[\operatorname{Sec}(X)] \leq m+x$. We will often use this fact to prove that iii) is not satisfied.

In this paper we give a precise description of all $x$-projectable Veronese varieties $X$ having only linear components (see Theorem 5), we prove that there are no 2-projectable varieties with $m \geq 3$ (see Theorem 4) and we give a complete classification of 3-projectable varieties by the following result.

Theorem 2. Let $X=X_{1} \cup X_{2} \cup \ldots \cup X_{r} \subset \mathbb{P}^{N}$ be a 3-projectable Veronese variety, according to Definition $3, \operatorname{dim}(X)=m \geq 3$. Then $\operatorname{dim}(X)=3$ and $X$ is one of the following:

1) $X \subset \mathbb{P}^{r+2}$ has only linear components, $r \geq 5$ and there exists a fixed component $X_{i}$ such that all other components intersect $X_{i}$ along planes of $X_{i}$ in general position;

2) $X \subset \mathbb{P}^{r+2}$ has only linear components, $r \geq 5$ and there are two fixed components $X_{i}$ and $X_{j}$ such that $X_{i} \cap X_{j}:=\Pi \simeq \mathbb{P}^{2}$ and all other components intersect $X_{i}$ 
along planes in general position, or intersect $X_{j}$ along planes in general position, and intersect each other along lines in general position in $\Pi$;

3) $X=X_{1} \cup X_{2} \subset \mathbb{P}^{7}, X_{1}$ is a cone of degree 3 having a line $E_{1}$ as vertex and a twisted cubic as base, $X_{2}$ is a cone of degree 2 having a point $E_{2}$ as vertex and $X_{1} \cap X_{2}$ is a plane containing $E_{1}$ and $E_{2}$ with $E_{1} \cap E_{2}=\emptyset$;

4) $X=X_{1} \cup X_{2} \cup X_{3} \subset \mathbb{P}^{7} ; X_{1}$ is a quadric cone having a line $E_{1}$ as vertex, $X_{2}$ is a quadric cone having a point $E_{2}$ as vertex, $X_{3} \simeq \mathbb{P}^{3} ; X_{1} \cap X_{2} \cap X_{3}$ is a plane $F$, the vertexes are disjoint (the role of $X_{2}$ and $X_{3}$ can be exchanged);

5) $X=X_{1} \cup X_{2} \cup X_{3} \subset \mathbb{P}^{7} ; X_{1}$ is a quadric cone having a line $E_{1}$ as vertex, $X_{2}$ is a quadric cone having a point $E_{2}$ as vertex, $X_{3} \simeq \mathbb{P}^{3} ; X_{1} \cap X_{2}$ is a plane $F$, $X_{3} \cap X_{1}$ is another plane $F^{\prime}, X_{3} \cap X_{2}=E_{1} \subset F$, the vertexes are disjoint (the role of $X_{2}$ and $X_{3}$ can be exchanged);

6) $X=X_{1} \cup X_{2} \cup \ldots \cup X_{r} \subset \mathbb{P}^{r+3}, r \geq 4 ; X_{1}$ is a quadric cone having a line $E_{1}$ as vertex, $X_{i} \simeq \mathbb{P}^{3}$ for $i \geq 2 ; X_{1} \cap X_{2}$ is a plane $F ; X_{i} \cap X_{2}$ are planes in generic position in $X_{2}$ intersecting lines $L_{i} \subset F$ in generic position for $i \geq 3$;

7) $X=X_{1} \cup X_{2} \cup \ldots \cup X_{r} \subset \mathbb{P}^{r+3}, r \geq 4 ; X_{1}$ is a quadric cone having a point $E_{1}$ as vertex, $X_{i} \simeq \mathbb{P}^{3}$ for $i \geq 2 ; X_{1} \cap X_{2}$ is a plane $F ; X_{i} \cap X_{2}$ are planes in generic position in $X_{2}$ intersecting lines $L_{i} \subset F$ in generic position for $i \geq 3$; possibly one of the components $X_{p}, 3 \leq p \leq r$, is exceptional: it intersects $X_{1}$ along another plane $F^{\prime}$, cutting $F$ along a line $l$ in generic position with respect to the set $\left\{L_{i}, i \neq p\right\}$

8) $X=X_{1} \cup X_{2} \cup \ldots \cup X_{r} \subset \mathbb{P}^{r+3}, r \geq 4 ; X_{1}$ is a quadric cone having a point $E_{1}$ as vertex, $X_{i} \simeq \mathbb{P}^{3}$ for $i \geq 2 ; X_{1} \cap X_{2}$ is a plane $F ; X_{i} \cap X_{2}$ are planes in generic position in $X_{2}$ intersecting lines $L_{i} \subset F$ in generic position for $i=3, \ldots, r-1$; there exists a fixed $j, 3 \leq j \leq r-1$, such that $X_{r} \cap X_{j}$ is a plane and $X_{r} \cap X_{1}=L_{j}$ $\left(E_{1} \notin L_{j}\right)$;

9) $X=X_{1} \cup X_{2} \cup X_{3} \cup X_{4} \subset \mathbb{P}^{7} ; X_{1}$ is a quadric cone having a point $E_{1}$ as vertex, $X_{i} \simeq \mathbb{P}^{3}$ for $i \geq 2 ; X_{1} \cap X_{2} \cap X_{3}$ is a plane $F ; X_{4} \cap X_{2}$ is a plane $\Pi$, $X_{4} \cap X_{3}=X_{4} \cap X_{1}$ is a line $L=\Pi \cap F$, not passing through $E_{1}$, or $X_{4} \cap X_{3}$ is a plane $\Pi, X_{4} \cap X_{2}=X_{4} \cap X_{1}$ is a line $L=\Pi \cap F$, not passing through $E_{1}$.

Our strategy will be: firstly to consider the case in which all components of $X$ are linear spaces, secondly to use Remark 2 to get a short list of possibilities, thirdly to prove (or disprove) that $X$ projects isomorphically by checking that the union of all Zariski tangent spaces at points $P \in X$ is disjoint with a general linear subspace of dimension $N-m-1-x$ of $\mathbb{P}^{N}$ (see Theorem 6.5 of [Ho], page 168, and Propositions 4.2 and 5.3 for the definiton of $Z \operatorname{arsec}(X, i)$, see also Corollary 2 of $[\mathrm{J}])$.

We will use the following definitions:

$\left\langle V_{1} \cup \ldots \cup V_{r}\right\rangle$ : linear span in $\mathbb{P}^{N}$ of the varieties $V_{i} \subset \mathbb{P}^{N}, i=1, \ldots, r$; when $V_{1}, \ldots, V_{r}$ are linear spaces we often write $V_{1} \cup \ldots \cup V_{r}$ instead of $\left\langle V_{1} \cup \ldots \cup V_{r}\right\rangle$;

$\operatorname{Sing}(V)$ : singular locus of the subscheme $V \subset \mathbb{P}^{N}$;

$T_{P}(V)$ : Zariski tangent space at a point $P$ of $V$; it is a projective subspace of $\mathbb{P}^{N}$ whose dimension is the embedding dimension of the local ring $\mathcal{O}_{V, P}$;

$\mathbb{T}_{P}$ : union of $T_{P}\left(V_{i}\right)$ for all irreducible components $V_{i} \subset V$ containing $P$; note that the linear span of $\mathbb{T}_{P}$ is always contained in $T_{P}(V)$ for any point $P \in V$;

$[V ; W]: \overline{\left\{\bigcup_{v \in V, w \in W, v \neq w}\langle v \cup w\rangle\right\}} \subset \mathbb{P}^{N}$, join of $V$ and $W$, for any pair of distinct irreducible varieties $V, W \subset \mathbb{P}^{N}$. In case $V=W,[V ; V]=\operatorname{Sec}(V)$ 
$\operatorname{Sec}(V): \overline{\left\{\bigcup_{v_{1} \neq v_{2} \in V}\left\langle v_{1} \cup v_{2}\right\rangle\right\}} \subset \mathbb{P}^{N}$ for any variety $V \subset \mathbb{P}^{N}$, if $V=V_{1} \cup \ldots \cup V_{r}$ is reducible then $\operatorname{Sec}(V)=\bigcup_{i, j=1, \ldots, r}\left[V_{i} ; V_{j}\right]$.

We will often use the following facts: if $V$ is an irreducible variety, not a linear space, for which there exists a linear space $L$, such that for any generic point $P \in V$, $T_{P}(V) \supseteq L$, then $V$ is a cone whose vertex contains $L$ (see [A2], page 17, but recall that, in our paper, a cone is never a linear space); if $V=V_{1} \cup \ldots \cup V_{r}$ is reducible and $x$-projectable then $\operatorname{dim}\left(\left[V_{i} ; V_{j}\right]\right) \leq m+x$ for any $i, j=1, \ldots, r$.

Let $a_{1} \geq a_{2} \geq \ldots \geq a_{k}>0$ be a set of integers, $k \geq 1$. Let us consider the rank $m \geq k$ vector bundle $\mathcal{E}:=\mathcal{O}_{\mathbb{P}^{1}}\left(a_{1}\right) \oplus \mathcal{O}_{\mathbb{P}^{1}}\left(a_{2}\right) \ldots \oplus \mathcal{O}_{\mathbb{P}^{1}}\left(a_{k}\right) \oplus \mathcal{O}_{\mathbb{P}^{1}} \ldots \oplus \oplus \mathcal{O}_{\mathbb{P}^{1}}$ over $\mathbb{P}^{1}$ and let $V$ be $\mathbb{P}(\mathcal{E})$. If $m=k \geq 2$, we say that $V$ is a smooth rational scroll of degree $\sum_{i=1}^{k} a_{i}$, embedded in a projective space of dimension $\sum_{i=1}^{k} a_{i}+k-1$ as a linearly normal variety. If $m>k \geq 2$, we say that $V$ is a cone over a smooth rational scroll, having a vertex $E$ of dimension $e:=m-k-1$; in this case $\operatorname{deg}(V)=$ $\sum_{i=1}^{k} a_{i}$, but $V$ is embedded in a projective space of dimension $\sum_{i=1}^{k} a_{i}+m-1$ as a linearly normal variety. If $m>k=1, a_{1} \geq 2$, we say that $V$ is a cone over $a$ rational normal curve of degree $a_{1}$, having a vertex $E$ of dimension $e=m-2$; in this case $\operatorname{deg}(V)=a_{1}$ and $V$ is embedded in a projective space of dimension $a_{1}$ $+m-1$ as a linearly normal variety. In all these cases $V$ is a variety of minimal degree, i.e. $\operatorname{deg}(V)=\operatorname{cod}(V)+1$, in its span which has always dimension $\sum_{i=1}^{k} a_{i}+$ $m-1$.

Acknowledgements: we wish to thank the referee for his very careful revision of the manuscript and for having pointed out a missing case in the first version.

\section{XAmbÓ's RESUlt AND FIRST REMARKS}

In Theorem 1 of $[\mathrm{X}]$ Xambó proves the following result:

Theorem 3. Let $V=V_{1} \cup \ldots . \cup V_{r} \subset \mathbb{P}^{N}$ be a non degenerate, reducible, reduced, variety of pure dimension $m \geq 2$, whose irreducible components are $V_{1}, \ldots, V_{r}$. Assume that $V$ is connected in codimension 1 and that it has minimal degree, then:

- any irreducible component $V_{i}$ of $V$ is an irreducible variety of dimension $m$ and minimal degree in its span $\left\langle V_{i}\right\rangle$;

- there is at least an ordering $V_{1}, V_{2}, \ldots, V_{r}$ such that, for any $j=2, \ldots, r$, $V_{j} \cap\left(V_{1} \cup \ldots \cup V_{j-1}\right)=\left\langle V_{j}\right\rangle \cap\left\langle V_{1} \cup \ldots \cup V_{j-1}\right\rangle$ and this intersection is always $a$ linear space of dimension exactly $m-1$.

From now on an ordering given by Theorem 3 will be called a good ordering.

Corollary 1. Let $V$ be any variety as in Theorem 3. Let $d_{i}$ be the degree of $V_{i}$. Then:

i) for any pair of irreducible components $V_{j}, V_{k} \subset V$ we have only three possibilities:

- $V_{j} \cap V_{k}=\left\langle V_{j}\right\rangle \cap\left\langle V_{k}\right\rangle=\emptyset$

- $V_{j} \cap V_{k}=\left\langle V_{j}\right\rangle \cap\left\langle V_{k}\right\rangle$ is a point

- $V_{j} \cap V_{k}=\left\langle V_{j}\right\rangle \cap\left\langle V_{k}\right\rangle$ is a linear space of dimension $\delta$ with $1 \leq \delta \leq m-1$; 
ii) for any $j \geq 2$, there is at least a component $V_{k}$ with $k<j$ such that $V_{j} \cap V_{k} \simeq$ $\mathbb{P}^{m-1}$ and the other intersections $V_{j} \cap V_{i}$, with $i<j$, are linear spaces contained in $V_{j} \cap V_{k}$, possibly coincident with it;

iii) $N=\operatorname{dim}(\langle V\rangle)=\sum_{i=1}^{r} \operatorname{dim}\left(\left\langle V_{i}\right\rangle\right)-(r-1)(m-1)=\sum_{i=1}^{r} d_{i}+m-1$;

iv) for any point $P \in V, T_{P}(V)=\left\langle\mathbb{T}_{P}\right\rangle$.

Proof. $i$ ) Let us assume that $V_{j} \cap V_{k} \neq \emptyset$ and that $k>j$ in a good ordering for the components of $V$. Then $V_{j} \cap V_{k} \subseteq V_{k} \cap\left(V_{1} \cup \ldots V_{j} \cup \ldots \cup V_{k-1}\right)$ which is a linear space of dimension $m-1$, as a scheme, because it is the intersection of two linear spaces in $\mathbb{P}^{N}$. By Theorem 0.4 of [E-G-H-P] $V$ is small according to the definition of [E-G-H-P], p.1364, hence $V_{j} \cap V_{k}=\left\langle V_{i}\right\rangle \cap\left\langle V_{j}\right\rangle$ is a linear space by Proposition 2.4 of [E-G-H-P]. As $V_{j} \cap V_{k}$ is contained in a linear space of dimension $m-1$, Corollary $1 i$ ) follows.

ii) By $i)$ we know that $V_{j} \cap\left(V_{1} \cup \ldots \cup V_{j-1}\right)=\left(V_{j} \cap V_{1}\right) \cup \ldots \cup\left(V_{j} \cap V_{j-1}\right)$ is the union of linear spaces of dimension $m-1$ at most. On the other hand we know that $V_{j} \cap\left(V_{1} \cup \ldots \cup V_{j-1}\right)$ is in fact a unique linear space of dimension exactly $m-1$ by Theorem 3 and $i$ i) follows.

iii) The first equality follows from the fact that, for any $j=2, \ldots, r, \operatorname{dim}\left(\left\langle V_{j}\right\rangle \cap\right.$ $\left.\left\langle V_{1} \cup \ldots \cup V_{j-1}\right\rangle\right)=m-1$; the second equality follows from the fact that $\operatorname{dim}\left(\left\langle V_{j}\right\rangle\right)=$ $m+d_{j}-1$ for any $j$.

$i v$ ) Recall that $\mathbb{T}_{P}$ is the union of the Zariski tangent spaces $T_{P}\left(V_{i}\right)$ for all irreducible components $V_{i} \subset V$ containing $P$. In our case $T_{P}(V)$ is the linear span of $\mathbb{T}_{P}$ thanks to property $i$ ).

By Theorem 3 it follows that any irreducible component of an $x$-projectable Veronese variety $X$ is an irreducible, $m$-dimensional variety of minimal degree in its span containing a linear space of dimension $m-1$. From the well known classification of such varieties (see for instance Theorem 0.1 of $[\mathrm{E}-\mathrm{G}-\mathrm{H}-\mathrm{P}]$ ) we have the following:

Corollary 2. Let $X_{i}$ be any irreducible component of an $x$-projectable Veronese variety $X \subset \mathbb{P}^{N}$, of dimension $m \geq 3$. Then, a priori, we have only the following possibilities:

i) $X_{i}$ is a linear space in $\mathbb{P}^{N}$ of dimension $m$;

ii) $X_{i}$ is a cone, having a vertex of dimension $e_{i} \geq 0$ over a smooth scroll having fibres of dimension $m-e_{i}-2 \geq 1$;

iii) $X_{i}$ is a cone, having a vertex of dimension $e_{i} \geq 0$ over a rational normal curve;

iv) $X_{i}$ is a smooth rational scroll and $m \leq x+1$.

Proof. By looking at Theorem 0.1 of [E-G-H-P], where irreducible, $m$-dimensional varieties of minimal degree in their spans are listed, we get $X_{i}$ may be a linear space or a hyperquadric, or as in $i i), i i i)$, or a smooth rational scroll, or a cone over a Veronese surface in $\mathbb{P}^{5}$. As $X_{i}$ must contain a linear space of dimension $m-1$ we can exclude cones over Veronese surfaces because such a surface does not contain lines. For the same reason we can exclude hyperquadrics of rank $\geq 5$; a hyperquadric of rank 4 is a cone over a smooth quadric of $\mathbb{P}^{3}$ which is a smooth rational scroll; a hyperquadric of rank 3 is a cone over a rational normal curve: a smooth conic. If $X_{i}$ is a smooth rational scroll its fibres of dimension $m-1$ are disjoint, so they have to remain disjoint after projecting $X$ in $\mathbb{P}^{m+x}$, but this is not possible if $m \geq x+2$. 
Proposition 1. Let $V$ be a variety as in Theorem 3.

i) Let $V_{i}$ be any component of $V$. Then there exists at least a good ordering for the $r$ components of $V$ such that $V_{i}=V_{1}$;

ii) Let $W=W_{1} \cup \ldots \cup W_{k} \subset V$ be the union of some components of $V, k<r$, such that the assumptions of Theorem 3 are true for $W$. Then there exists at least a good ordering for the $r$ components of $V$ such that $V_{i}=W_{i}$ for $i=1, \ldots, k$.

Proof. $i$ ) Let $W \subset V$ be a proper subvariety of $V$ such that $W=V_{1} \cup \ldots \cup V_{\rho}$ with $1 \leq \rho<r$. Let us assume that $W$ is connected in codimension 1 . Then we claim the existence of at least a component $V_{i} \subset V$ such that $\operatorname{dim}\left(W \cap V_{i}\right)=m-1$ and $W \cup V_{i}$ is connected in codimension 1 .

In fact, if $\operatorname{dim}\left(W \cap V_{i}\right) \leq m-2$ for any irreducible component $V_{i} \subset V$ with $\rho<i \leq r$, then $\operatorname{dim}\left[W \cap\left(V_{\rho+1} \cup \ldots \cup V_{r}\right)\right] \leq m-2$, but this is not possible, otherwise $V \backslash\left[W \cap\left(V_{\rho+1} \cup \ldots \cup V_{r}\right)\right]$ would be not connected while we are assuming that $V$ is connected in codimension 1. Hence, by changing the ordering of $V_{\rho+1}, \ldots, V_{r}$ if necessary, we can assume that $\operatorname{dim}\left(W \cap V_{\rho+1}\right) \geq m-1$. It is not possible that $\operatorname{dim}\left(W \cap V_{\rho+1}\right) \geq m$, otherwise the irreducible surface $V_{\rho+1}$ would be a component of $W$, so that $\operatorname{dim}\left(W \cap V_{\rho+1}\right)=m-1$. Let us consider $W \cup V_{\rho+1} . W$ is connected in codimension 1 by assumptions, $V_{\rho+1}$ is connected in codimension 1 because it is an irreducible variety of dimension $m$; as $\operatorname{dim}\left(W \cap V_{\rho+1}\right)=m-1$ we have $W \cup V_{\rho+1}$ is connected in codimension 1 too.

Now, let us choose a component $V^{\prime} \subset V$ and let us consider a good ordering. If $V^{\prime}=V_{1}$ we have nothing to prove, otherwise, in any case, there exists at least another component $V_{j}$ such that $V^{\prime} \cap V_{j} \simeq \mathbb{P}^{m-1}$. Let us prove that there exists another good ordering having $V^{\prime}$ at the first position. Set $V_{1}=V^{\prime}$ and $V_{2}=V_{j}$. If $r=2$ we are done, if not we can apply the above remark with $W=V_{1} \cup V_{2}$ and we get another component $V_{3}$ such that $\operatorname{dim}\left(W \cap V_{3}\right)=m-1$ and $W \cup V_{3}$ is connected in codimension 1. By applying the above remark a suitable number of times we get an ordering $V_{1}, \ldots, V_{r}$ such that $V_{1}=V^{\prime}, \operatorname{dim}\left[V_{j} \cap\left(V_{1} \cup \ldots \cup V_{j-1}\right)\right]=m-1$ for any $j \geq 2$, and $V_{1} \cup \ldots \cup V_{j}$ is connected in codimension 1 . As $V_{j} \cap\left(V_{1} \cup \ldots \cup V_{j-1}\right) \subseteq$ $\left(\left\langle V_{j}\right\rangle \cap\left\langle V_{1} \cup \ldots \cup V_{j-1}\right\rangle\right)$ for any $j \geq 2$, we have only to prove that $\operatorname{dim}\left(\left\langle V_{j}\right\rangle \cap\right.$ $\left.\left\langle V_{1} \cup \ldots \cup V_{j-1}\right\rangle\right)=m-1$ to get $V_{j} \cap\left(V_{1} \cup \ldots \cup V_{j-1}\right)=\left\langle V_{j}\right\rangle \cap\left\langle V_{1} \cup \ldots \cup V_{j-1}\right\rangle$ $\simeq \mathbb{P}^{m-1}$ for any $j \geq 2$, hence to prove the Proposition.

Let us put $\operatorname{deg}\left(V_{i}\right)=d_{i}$; obviously $\operatorname{dim}\left(\left\langle V_{i}\right\rangle\right)=m-1+d_{i}$.

Let us put $a_{j}:=\operatorname{dim}\left(\left\langle V_{j}\right\rangle \cap\left\langle V_{1} \cup \ldots \cup V_{j-1}\right\rangle\right)$ for any $j \geq 2$, so that:

$\operatorname{dim}\left(\left\langle V_{1} \cup V_{2}\right\rangle\right)=m-1+d_{1}+m-1+d_{2}-a_{2}$

$\operatorname{dim}\left(\left\langle V_{1} \cup V_{2} \cup V_{3}\right\rangle\right)=\operatorname{dim}\left(\left\langle\left\langle V_{1} \cup V_{2}\right\rangle \cup\left\langle V_{3}\right\rangle\right\rangle=\right.$

$=m-1+d_{1}+m-1+d_{2}-a_{2}+m-1+d_{3}-a_{3}$

$\operatorname{dim}\left(\left\langle V_{1} \cup V_{2} \cup \ldots \cup V_{r}\right\rangle\right)=r(m-1)+\sum_{j=1}^{r} d_{j}-\sum_{j=2}^{r} a_{j}=N$.

On the other hand: $\operatorname{dim}(\langle V\rangle)=N=m-1+\operatorname{deg}(V)=m-1+\sum_{j=1}^{r} d_{j}$ so that we have: $r(m-1)-\sum_{j=2}^{r} a_{j}=m-1$, i.e. $(r-1)(m-1)=\sum_{j=2}^{r} a_{j}$.

As $a_{j} \geq m-1$ for any $j \geq 2$ we get $a_{j}=m-1$ for any $j \geq 2$ and we are done.

ii) As in the proof of $i$ ) we can do induction, starting from $W$ instead of $V^{\prime}=V_{1}$, knowing that $a_{j}=m-1$ for $2 \leq j \leq k$. 
Let us recall the Terracini's lemma:

Lemma 1. Let us consider a pair of irreducible varieties $V, W \subset \mathbb{P}^{N}$ and a generic point $R \in[V ; W]$ such that $R \in\langle P \cup Q\rangle$, with $P \in V$ and $Q \in W$, (hence $P$ and $Q$ are generic points of $V$ and $W$, respectively), then $T_{R}([V ; W])=\left\langle T_{P}(V) \cup T_{Q}(W)\right\rangle$ and $\operatorname{dim}([V ; W])=\operatorname{dim}\left(\left\langle T_{P}(V) \cup T_{Q}(W)\right\rangle\right)$.

Proof. See Corollary 1.11 of [A1].

Corollary 3. Let $V, W \subset \mathbb{P}^{N}$ be two irreducible varieties such that $V \cap W=\emptyset$, then $\operatorname{dim}([V ; W])=\operatorname{dim}(V)+\operatorname{dim}(W)+1$.

Proof. Obviously $\operatorname{dim}([V ; W]) \leq \operatorname{dim}(V)+\operatorname{dim}(W)+1$; if $V \cap W=\emptyset$ we have $\operatorname{dim}([V ; W]) \geq \operatorname{dim}(V)+\operatorname{dim}(W)+1$, see for instance Corollary 2.5 of $[\mathrm{A} 1]$.

Lemma 2. Let $X \subset \mathbb{P}^{N}$ be an $x$-projectable Veronese variety. Then:

i) any irreducible component $X_{i} \subset X$ can be isomorphically projected in $\mathbb{P}^{m+x}$;

ii) for any pair of irreducible components $X_{j}$ and $X_{k}$ of $X$ we have $\operatorname{dim}\left(X_{j} \cap\right.$ $\left.X_{k}\right) \geq m-x$, and, unless $X_{j}$ and $X_{k}$ are both linear spaces, $\operatorname{dim}\left(X_{j} \cap X_{k}\right) \geq$ $m-x+1$;

iii) for any irreducible, not linear, component $X_{i} \subset X$ let $Y_{i}$ be any linear space of maximal dimension $m-1$ contained in $X_{i}$, then $Y_{j} \cap Y_{k} \neq \emptyset$ if $m \geq x+2$ and $X_{j} \cap Y_{k} \neq \emptyset$ if $m \geq x+1$.

Proof. i) Obvious.

ii) Since $X$ is an $x$-projectable Veronese variety, there exists a projection $\pi_{\mathcal{L}}$ : $\mathbb{P}^{N}--->\Lambda$ from a suitable linear space $\mathcal{L}$ to a suitable linear space $\Lambda \subset \mathbb{P}^{N}, \Lambda \simeq$ $\mathbb{P}^{m+x}$, such that $\pi_{\mathcal{L}}(X) \simeq X$. This implies that, for any $i=1, \ldots, r, \pi_{\mathcal{L}}\left(X_{i}\right) \simeq X_{i}$, and, for any pair $X_{j}, X_{k} \subset X, \pi_{\mathcal{L}}\left(X_{j} \cap X_{k}\right) \simeq \pi_{\mathcal{L}}\left(X_{j}\right) \cap \pi_{\mathcal{L}}\left(X_{k}\right) \simeq X_{j} \cap X_{k}=\left\langle X_{j}\right\rangle \cap$ $\left\langle X_{k}\right\rangle$ is a linear space by Corollary 1 . As $\pi_{\mathcal{L}}\left(X_{j}\right)$ and $\pi_{\mathcal{L}}\left(X_{k}\right)$ are two irreducible varieties of dimension $m$ in $\mathbb{P}^{m+x}$ we have $\operatorname{dim}\left[\pi_{\mathcal{L}}\left(X_{j}\right) \cap \pi_{\mathcal{L}}\left(X_{k}\right)\right] \geq m-x$. If $\operatorname{dim}\left[\pi_{\mathcal{L}}\left(X_{j}\right) \cap \pi_{\mathcal{L}}\left(X_{k}\right)\right]=m-x$ we have two irreducible varieties of dimension $m$ in $\mathbb{P}^{m+x}$ whose scheme-theoretic intersection is a linear space of dimension $m-x$. By Bezout's theorem this is possibly only if they are both linear spaces.

iii) Let us consider any pair of linear spaces $Y_{j}$ and $Y_{k}$. By contradiction let us suppose that $Y_{j} \cap Y_{k}=\emptyset$, then, by Corollary 3 , their join has dimension $m-1+$ $m-1+1=2 m-1 \leq m+x$, because $\operatorname{dim}[\operatorname{Sec}(X)] \leq m+x$, and this is not possible if $m \geq x+2$. Let us consider any pair $X_{j}$ and $Y_{k}$. By contradiction let us suppose that $X_{j} \cap Y_{k}=\emptyset$, then, by Corollary 3 , their join has dimension $m+m-1+1=2 m$ $\leq m+x$, because $\operatorname{dim}[\operatorname{Sec}(X)] \leq m+x$, and this is not possible if $m \geq x+1$.

Lemma 3. Let $X \subset \mathbb{P}^{N}$ be an $x$-projectable Veronese variety. Let $P$ be a singular point of $X$ and let $X_{1}^{P}, \ldots, X_{s}^{P}$ be the irreducible components of $X$ containing $P$ with $s \geq 2$. For any $i=1, \ldots, s$ let $T_{i}$ be the tangent space of $X_{i}^{P}$ at $P$ in $\left\langle X_{i}^{P}\right\rangle$ and let us assume that the natural ordering of $X_{1}^{P}, \ldots, X_{s}^{P}$ is coherent with a good ordering. Then, for any $j=2, \ldots, s, T_{j} \nsubseteq\left\langle T_{1} \cup \ldots \cup T_{j-1}\right\rangle$ and $\operatorname{dim}\left[T_{j} \cap\left\langle T_{1} \cup \ldots \cup T_{j-1}\right\rangle\right] \leq$ $m-1$.

Proof. By contradiction, let us assume that $T_{j} \subseteq\left\langle T_{1} \cup \ldots \cup T_{j-1}\right\rangle$, hence $T_{j} \subseteq T_{j} \cap$ $\left\langle T_{1} \cup \ldots \cup T_{j-1}\right\rangle \subseteq\left\langle X_{j}^{P}\right\rangle \cap\left\langle X_{1}^{P} \cup \ldots \cup X_{j-1}^{P}\right\rangle$. As we are assuming that the natural ordering of $X_{1}^{P}, \ldots, X_{s}^{P}$ is coherent with a good ordering, we have $\operatorname{dim}\left[\left\langle X_{j}^{P}\right\rangle \cap\right.$ $\left.\left\langle X_{1}^{P} \cup \ldots \cup X_{j-1}^{P}\right\rangle\right] \leq m-1$. Moreover $\operatorname{dim}\left(T_{j}\right)=m$ if $P$ is a smooth point of $X_{j}^{P}$ 
and $\operatorname{dim}\left(T_{j}\right) \geq m+1$ if $P$ is a singular point of $X_{j}^{P}$. So that in any case we get a contradiction. By the way we have also proved that $\operatorname{dim}\left[T_{j} \cap\left\langle T_{1} \cup \ldots \cup T_{j-1}\right\rangle\right] \leq$ $m-1$.

Lemma 4. Let $X \subset \mathbb{P}^{N}$ be an $x$-projectable Veronese variety. Let $P$ be any point of $X$ and let $X_{1}^{P}, \ldots, X_{s}^{P}$ be the irreducible components of $X$ containing $P, s \geq 1$. For any $i=1, \ldots, s$ let $T_{i}$ be the Zariski tangent space of $X_{i}^{P}$ at $P$ in $\left\langle X_{i}^{P}\right\rangle$ and let us define $\mathbb{T}_{P}:=\bigcup_{i=1}^{s} T_{i}$. Then $\operatorname{dim}\left(\left\langle\mathbb{T}_{P}\right\rangle\right) \leq m+x$. Moreover, if $P \in \operatorname{Sing}\left(X_{i}\right)$ $T_{i}=\left\langle X_{i}\right\rangle$ and, for any $P \in X$, we have $T_{P}(X)=\left\langle\mathbb{T}_{P}\right\rangle$.

Proof. If $s=1$ we have $\left\langle\mathbb{T}_{P}\right\rangle=T_{1}$ and $\operatorname{dim}\left(T_{1}\right) \leq m+x$ by Lemma $2 i$ ). If $s \geq 2, \mathbb{T}_{P}$ is the union of $s$ linear spaces, of dimensions $\geq m$, passing through $P$ according a certain configuration $\mathcal{C}_{P} \subset \mathbb{P}^{N}$. By contradiction, let us assume that $\operatorname{dim}\left(\left\langle\mathbb{T}_{P}\right\rangle\right) \geq m+x+1$. Let $\pi_{\mathcal{L}}: \mathbb{P}^{N}-->\Lambda$ be any linear projection, from a suitable $(N-m-1-x)$-dimensional linear space $\mathcal{L}$ to a suitable $\Lambda \subset \mathbb{P}^{N}, \Lambda \simeq$ $\mathbb{P}^{m+x}$, such that $\pi_{\mathcal{L}}(X)$ is isomorphic to $X$, hence $\pi_{\mathcal{L}}\left(\mathcal{C}_{P}\right)$ is isomorphic to $\mathcal{C}_{P}$. As $\operatorname{dim}\left(\left\langle\mathbb{T}_{P}\right\rangle\right) \geq m+x+1$ there is a non empty linear space $\mathcal{L}^{\prime}:=\mathcal{L} \cap\left\langle\mathbb{T}_{P}\right\rangle$ such that $\pi_{\mathcal{L}}\left(\mathcal{C}_{P}\right)=\pi_{\mathcal{L}^{\prime}}\left(\mathcal{C}_{P}\right)$ where $\pi_{\mathcal{L}^{\prime}}:\left\langle\mathbb{T}_{P}\right\rangle-->\Lambda$. But, as $\operatorname{dim}(\Lambda)<\operatorname{dim}\left(\left\langle\mathbb{T}_{P}\right\rangle\right)$, it is not possible that $\pi_{\mathcal{L}^{\prime}}\left(\mathcal{C}_{P}\right) \simeq \mathcal{C}_{P}$, otherwise isomorphic configurations of linear spaces would have linear spans of different dimensions, so that we get a contradiction.

Now, by Corollary 2, we have $T_{i}=\left\langle X_{i}\right\rangle$ if $P \in \operatorname{Sing}\left(X_{i}\right)$ and, by Corollary $1 \mathrm{iv}$ ) the Zariski tangent space $T_{P}(X)$ is exactly $\left\langle\mathbb{T}_{P}\right\rangle$ for any point $P \in X$.

Corollary 4. Let $X \subset \mathbb{P}^{N}$ be an $x$-projectable Veronese variety, $\operatorname{dim}(X)=m \geq 3$. Let $P$ be any point of $X$. Then there are at most $x+1$ irreducible components of $X$ passing through $P$.

Proof. Let us assume that there are at least two irreducible components of $X$ passing through $P$, so that $P \in \operatorname{Sing}(X)$. We know that $\operatorname{dim}\left(\left\langle\mathbb{T}_{P}\right\rangle\right) \leq m+x$ by Lemma 4 , on the other hand we can apply Lemma 3 to the set $\left\{X_{1}^{P}, \ldots, X_{s}^{P}\right\}$ of irreducible components containing $P$ and we have $\operatorname{dim}\left(T_{1}\right) \geq m$ and $\operatorname{dim}\left(\left\langle T_{1} \cup \ldots \cup T_{j}\right\rangle\right)$ increases of a unity at least for $j=2, \ldots, s$. If $s \geq x+2$ we would have $\operatorname{dim}\left(\left\langle\mathbb{T}_{P}\right\rangle\right)=$ $\operatorname{dim}\left(\left\langle T_{1} \cup \ldots \cup T_{s}\right\rangle\right) \geq m+s-1 \geq m+x+1$, contradiction.

Proposition 2. Let $X \subset \mathbb{P}^{N}$ be an $x$-projectable Veronese variety, $\operatorname{dim}(X)=m \geq$ 3. Let $X_{i}$ be an irreducible component of $X$, of degree $d_{i}$, which is neither a linear space nor a smooth scroll, then $X_{i}=\mathbb{P}(\mathcal{E})$ where $\mathcal{E}$ is a vector bundle over $\mathbb{P}^{1}$ of the following type:

$\mathcal{O}_{\mathbb{P}^{1}}\left(a_{1}\right) \oplus \ldots \oplus \mathcal{O}_{\mathbb{P}^{1}}\left(a_{k_{i}}\right) \oplus \mathcal{O}_{\mathbb{P}^{1}} \ldots \oplus \oplus \mathcal{O}_{\mathbb{P}^{1}}, X_{i} \subset \mathbb{P}^{m+d_{i}-1}, d_{i}=\sum_{j=1}^{k_{i}} a_{j} \leq x$.

If $x=3$ we have only the following possibilities:

a) $\mathcal{O}_{\mathbb{P}^{1}}(1) \oplus \mathcal{O}_{\mathbb{P}^{1}}(1) \oplus \mathcal{O}_{\mathbb{P}^{1}}(1) \oplus \mathcal{O}_{\mathbb{P}^{1}} \ldots \oplus \mathcal{O}_{\mathbb{P}^{1}}, X_{i} \subset \mathbb{P}^{m+2}, d_{i}=3$

b) $\mathcal{O}_{\mathbb{P}^{1}}(2) \oplus \mathcal{O}_{\mathbb{P}^{1}}(1) \oplus \mathcal{O}_{\mathbb{P}^{1}} \ldots \oplus \mathcal{O}_{\mathbb{P}^{1}}, X_{i} \subset \mathbb{P}^{m+2}, d_{i}=3$

c) $\mathcal{O}_{\mathbb{P}^{1}}(3) \oplus \mathcal{O}_{\mathbb{P}^{1}} \ldots \oplus \mathcal{O}_{\mathbb{P}^{1}}, X_{i} \subset \mathbb{P}^{m+2}, d_{i}=3$

d) $\mathcal{O}_{\mathbb{P}^{1}}(1) \oplus \mathcal{O}_{\mathbb{P}^{1}}(1) \oplus \mathcal{O}_{\mathbb{P}^{1}} \ldots \oplus \mathcal{O}_{\mathbb{P}^{1}}, X_{i} \subset \mathbb{P}^{m+1}, d_{i}=2$

e) $\mathcal{O}_{\mathbb{P}^{1}}(2) \oplus \mathcal{O}_{\mathbb{P}^{1}} \ldots \oplus \mathcal{O}_{\mathbb{P}^{1}}, X_{i} \subset \mathbb{P}^{m+1}, d_{i}=2$.

The above cones have a vertex $E_{i}$ of dimension $e_{i}=m-k_{i}-1 \geq 0$ and none of them can be isomorphically projected into a linear space unless $\operatorname{dim}\left(\left\langle X_{i}\right\rangle\right)=$ $m+d_{i}-1 \leq m+x-1$ and the linear span of the cone is isomorphically projected. 
Proof. Let us fix a good ordering among the irreducible components of $X$; we know that $X_{i}$ is a variety of minimal degree in its span. Let us assume that $X_{i}$ is a cone as in $i$ ) or $i$ ii) of Corollary 2 and let $P$ be a point in $\operatorname{Sing}\left(X_{i}\right)=E_{i}$. By Lemma 4 we know that $\operatorname{dim}\left[T_{P}\left(X_{i}\right)\right] \leq m+x$, hence $\operatorname{dim}\left\langle X_{i}\right\rangle=\operatorname{dim}\left[T_{P}\left(X_{i}\right)\right] \leq m+x$.

By contradiction, let us assume that $\operatorname{dim}\left\langle X_{i}\right\rangle=\operatorname{dim}\left[T_{P}\left(X_{i}\right)\right]=m+x$. If $i \neq 1$ we have $\left\langle X_{1} \cup \ldots \cup X_{i-1}\right\rangle \cap\left\langle X_{i}\right\rangle=\left(X_{1} \cup \ldots \cup X_{i-1}\right) \cap X_{i} \simeq \mathbb{P}^{m-1}$ and we can choose a component $X_{j}$, with $j<i$, such that $X_{j} \cap X_{i}=\left\langle X_{j}\right\rangle \cap\left\langle X_{i}\right\rangle \simeq \mathbb{P}^{m-1}$. If $i=1$ let us consider $X_{2}$ and we have $X_{1} \cap X_{2}=\left\langle X_{1}\right\rangle \cap\left\langle X_{2}\right\rangle \simeq \mathbb{P}^{m-1}$. In any case we can find another component $X_{j}$ of $X$ such that $X_{j} \cap X_{i}=\left\langle X_{j}\right\rangle \cap\left\langle X_{i}\right\rangle \simeq \mathbb{P}^{m-1}$. Note that $P \in X_{j}$ because $E_{i}$ is contained in all $(m-1)$-dimensional linear spaces contained in $X_{i}$ and one of them is containd also in $X_{j}$. Let us consider $T_{P}\left(X_{j}\right) \cup T_{P}\left(X_{i}\right)$, by Lemma 4 we know that $\operatorname{dim}\left[T_{P}\left(X_{j}\right) \cup T_{P}\left(X_{i}\right)\right] \leq m+x$, hence $T_{P}\left(X_{j}\right) \subseteq T_{P}\left(X_{i}\right)=$ $\left\langle X_{i}\right\rangle$, so that $T_{P}\left(X_{j}\right) \subseteq\left\langle X_{j}\right\rangle \cap\left\langle X_{i}\right\rangle$. As $\operatorname{dim}\left[T_{P}\left(X_{j}\right)\right] \geq m$ this is a contradiction.

The conclusion is that $\operatorname{dim}\left\langle X_{i}\right\rangle=\operatorname{dim}\left[T_{P}\left(X_{i}\right)\right] \leq m+x-1$. Now let us recall that for a cone as $i$ ) or $i$ ii) of Corollary 2 the linear span has dimension: $\sum_{j=1}^{k} a_{j}+$ $m-1=d_{i}+m-1$. Hence we get $d_{i}+m-1 \leq m+x-1$ and $d_{i} \leq x$. It is easy to see that if $x=3$ the only possibilities are the above ones.

Let $X_{i}$ be a cone as above, over a smooth base $B_{i}$ and having a vertex $E_{i}$. Note that $E_{i} \cap\left\langle B_{i}\right\rangle=\emptyset ; \operatorname{dim}\left(X_{i}\right)=\operatorname{dim}\left(B_{i}\right)+\operatorname{dim}\left(E_{i}\right)+1 ; \operatorname{dim}\left(\left\langle X_{i}\right\rangle\right)=\operatorname{dim}\left\langle B_{i}\right\rangle+$ $\operatorname{dim}\left(E_{i}\right)+1$. Hence $\operatorname{Sec}\left(X_{i}\right)$ contains the cone over $\operatorname{Sec}\left(B_{i}\right)$ having vertex $E_{i}$ and $\operatorname{dim}\left[\operatorname{Sec}\left(X_{i}\right)\right] \geq \operatorname{dim}\left[\operatorname{Sec}\left(B_{i}\right)\right]+\operatorname{dim}\left(E_{i}\right)+1$. Therefore $X_{i}$ can be isomorphically projected into a linear space of dimension smaller than the dimension of its linear span (i.e. $\operatorname{dim}\left[\operatorname{Sec}\left(X_{i}\right)<\operatorname{dim}\left(\left\langle X_{i}\right\rangle\right)\right)$ only if this is true also for $B_{i}$ (i.e. $\operatorname{dim}\left[\operatorname{Sec}\left(B_{i}\right)\right]<\operatorname{dim}\left(\left\langle B_{i}\right\rangle\right)$. By the well known classification of smooth irreducible varieties of small degree, which are all projectively normal, it follows that this is not possible, so that this is not possible for every $X_{i}$ too.

Proposition 3. Let $X \subset \mathbb{P}^{N}$ be an $x$-projectable Veronese variety, $\operatorname{dim}(X)=m \geq$ 3. Let $X_{i}$ be an irreducible component of $X$ which is a smooth scroll of degree $d_{i}$, then $X_{i}=\mathbb{P}(\mathcal{E})$ where $\mathcal{E}$ is a vector bundle over $\mathbb{P}^{1}$ of the following type:

$$
\mathcal{O}_{\mathbb{P}^{1}}\left(a_{1}\right) \oplus \ldots \oplus \mathcal{O}_{\mathbb{P}^{1}}\left(a_{m}\right), X_{i} \subset \mathbb{P}^{m+d_{i}-1}, \sum_{j=1}^{m} a_{j}-1=d_{i}-1 \leq x, m \leq x+1 .
$$

If $x=3$ we have only the following possibilities:

$\mathcal{O}_{\mathbb{P}^{1}}(2) \oplus \mathcal{O}_{\mathbb{P}^{1}}(1) \oplus \mathcal{O}_{\mathbb{P}^{1}}(1), X_{i} \subset \mathbb{P}^{6}, m=3, \operatorname{deg}\left(X_{i}\right)=4$

$\mathcal{O}_{\mathbb{P}^{1}}(1) \oplus \mathcal{O}_{\mathbb{P}^{1}}(1) \oplus \mathcal{O}_{\mathbb{P}^{1}}(1), X_{i} \subset \mathbb{P}^{5}, m=3, \operatorname{deg}\left(X_{i}\right)=3$.

None of the above scrolls can be isomorphically projected into a linear space unless the linear span of the scroll is isomorphically projected.

Proof. By Corollary $2 i v), m \leq x+1$. By Lemma $2 i$ ) we know that $X_{i}$ must be isomorphically projected into $\mathbb{P}^{m+x}$, but $\operatorname{dim}\left[\operatorname{Sec}\left(X_{i}\right)\right]=\min \left\{\operatorname{dim}\left(\left\langle X_{i}\right\rangle\right), 2 m+1\right\}$ (see [C]), hence it must be $\min \left\{\operatorname{dim}\left(\left\langle X_{i}\right\rangle\right), 2 m+1\right\} \leq m+x$. As $2 m+1>m+x$, it must be $\operatorname{dim}\left(\left\langle X_{i}\right\rangle\right) \leq m+x$. As in the proof of Proposition $2 \operatorname{dim}\left(\left\langle X_{i}\right\rangle\right)=\sum_{j=1}^{m} a_{j}$ $+m-1=d_{i}+m-1$, hence $d_{i}+m-1 \leq m+x$.

If $x=3$ it is easy to see that the only possibilities are the above ones and the following one: $\mathcal{O}_{\mathbb{P}^{1}}(1) \oplus \mathcal{O}_{\mathbb{P}^{1}}(1) \oplus \mathcal{O}_{\mathbb{P}^{1}}(1) \oplus \mathcal{O}_{\mathbb{P}^{1}}(1), X_{i} \subset \mathbb{P}^{7}, m=4, \operatorname{deg}\left(X_{i}\right)=4$.

However this last possibility cannot occur. By contradiction, let us assume that $X$ has a component as above. By Proposition 1 we can assume that the component 
is $X_{1}$, note that $X_{1} \simeq \mathbb{P}^{1} \times \mathbb{P}^{3}$. Let $Z_{1 j}$ be the linear space which is the intersection $X_{1} \cap X_{j}=\left\langle X_{1}\right\rangle \cap\left\langle X_{j}\right\rangle$ for $j \geq 2$. By Lemma $\left.2 i i\right), \operatorname{dim}\left(Z_{1 j}\right) \in\{3,2\}$ and, by Lemma 2 iii), $Z_{1 j}$ must intersect any linear space of dimension 3 contained in $X_{1}$. But it is easy to see that in $\mathbb{P}^{1} \times \mathbb{P}^{3} \subset \mathbb{P}^{7}$ all linear spaces of dimension 2 and 3 are contained in a unique linear space of dimension 3.

Corollary 5. Let $X \subset \mathbb{P}^{N}$ be an $x$-projectable Veronese variety, $\operatorname{dim}(X)=m \geq 3$. Let us assume that $X$ contains an irreducible component $X_{i}$ which is a cone of degree $d_{i}$, having a vertex $E_{i}$ of dimension $e_{i} \geq 0$. Let $Z_{i j}$ be the linear space which is the intersection $X_{i} \cap X_{j}=\left\langle X_{i}\right\rangle \cap\left\langle X_{j}\right\rangle$ for $j \neq i$, then $\operatorname{dim}\left(E_{i} \cap Z_{i j}\right) \geq e_{i}-x+2$. When $x=3$ this fact implies:

a) if $X_{i}$ is a cone of degree $3, e_{i}=m-4$ (necessarily $m \geq 4$ ) $Z_{i j} \cap E_{i} \neq \emptyset$;

b) if $X_{i}$ is a cone of degree $3, e_{i}=m-3, Z_{i j} \cap E_{i} \neq \emptyset$ when $m \geq 4$;

c) if $X_{i}$ is a cone of degree $3, e_{i}=m-2, Z_{i j} \cap E_{i} \neq \emptyset$;

d) if $X_{i}$ is a cone of degree $2, e_{i}=m-3, Z_{i j} \cap E_{i} \neq \emptyset$ when $m \geq 4$;

e) if $X_{i}$ is a cone of degree $2, e_{i}=m-2, Z_{i j} \cap E_{i} \neq \emptyset$.

Proof. By Corollary 1 and Lemma 2 ii $)$ we know that $m-1 \geq \operatorname{dim}\left(Z_{i j}\right) \geq m-x+1$. If $\operatorname{dim}\left(Z_{i j}\right)=m-1$ then $Z_{i j}$ contains the vertex $E_{i}$ of $X_{i}$ and we have nothing to prove. Let us assume that $m-2 \geq \operatorname{dim}\left(Z_{i j}\right)$. Let $B_{i}$ be the base of the cone $X_{i}$. Note that $E_{i} \cap\left\langle B_{i}\right\rangle=\emptyset$ and that $X_{i}=\left[E_{i} ; B_{i}\right]$, hence $\left\langle X_{i}\right\rangle=\left\langle\left[E_{i} ; B_{i}\right]\right\rangle=\left\langle E_{i} \cup\left\langle B_{i}\right\rangle\right\rangle$, moreover if we project $Z_{i j}$ from $E_{i}$ onto $\left\langle B_{i}\right\rangle$ we get in fact a linear space of $B_{i}$ having dimension $\operatorname{dim}\left[\left(E_{i} \cup Z_{i j}\right) \cap\left\langle B_{i}\right\rangle\right]$. Recall that the linear spaces contained in $B_{i}$ have dimension $\operatorname{dim}\left(B_{i}\right)-1$ at most, hence $\operatorname{dim}\left[\left(E_{i} \cup Z_{i j}\right) \cap\left\langle B_{i}\right\rangle\right] \leq \operatorname{dim}\left(B_{i}\right)-1$. Obviously:

$\operatorname{dim}\left(E_{i} \cup Z_{i j}\right)=e_{i}+\operatorname{dim}\left(Z_{i j}\right)-\operatorname{dim}\left(E_{i} \cap Z_{i j}\right) \geq e_{i}+m-x+1-\operatorname{dim}\left(E_{i} \cap Z_{i j}\right)$ $\operatorname{dim}\left(\left\langle X_{i}\right\rangle\right)=\operatorname{dim}\left(\left\langle E_{i} \cup\left\langle B_{i}\right\rangle\right\rangle\right)=\operatorname{dim}\left(\left\langle E_{i} \cup Z_{i j} \cup\left\langle B_{i}\right\rangle\right\rangle\right)=\operatorname{dim}\left(E_{i} \cup Z_{i j} \cup\left\langle B_{i}\right\rangle\right)$ $\operatorname{dim}\left(E_{i} \cup Z_{i j} \cup\left\langle B_{i}\right\rangle\right)=\operatorname{dim}\left(E_{i} \cup Z_{i j}\right)+\operatorname{dim}\left(\left\langle B_{i}\right\rangle\right)-\operatorname{dim}\left[\left(E_{i} \cup Z_{i j}\right) \cap\left\langle B_{i}\right\rangle\right]$, hence:

$$
\begin{aligned}
& \operatorname{dim}\left[\left(E_{i} \cup Z_{i j}\right) \cap\left\langle B_{i}\right\rangle\right] \geq e_{i}+m-x+1-\operatorname{dim}\left(E_{i} \cap Z_{i j}\right)+\operatorname{dim}\left(\left\langle B_{i}\right\rangle\right)-\operatorname{dim}\left(\left\langle X_{i}\right\rangle\right) \\
& e_{i}+m-x+1-\operatorname{dim}\left(E_{i} \cap Z_{i j}\right)+\operatorname{dim}\left(\left\langle B_{i}\right\rangle\right)-\operatorname{dim}\left(\left\langle X_{i}\right\rangle\right) \leq \operatorname{dim}\left(B_{i}\right)-1 \\
& e_{i}+m-x+1-\operatorname{dim}\left(E_{i} \cap Z_{i j}\right)+\left(d_{i}+\operatorname{dim}\left(B_{i}\right)-1\right)-\left(m+d_{i}-1\right) \leq \operatorname{dim}\left(B_{i}\right)-1 \\
& e_{i}-x+2 \leq \operatorname{dim}\left(E_{i} \cap Z_{i j}\right) .
\end{aligned}
$$

When $x=\overline{3}, \operatorname{dim}\left(E_{i} \cap Z_{i j}\right) \geq e_{i}-1$ and we have only the five possibilities listed by Proposition 2 .

a) In this case $e_{i}=m-4 \geq 0$, hence $\operatorname{dim}\left(E_{i} \cap Z_{i j}\right) \geq m-5 \geq 0$ if $m \geq 5$. When $m=4$, $\operatorname{dim}\left(E_{i}\right)=0$ and $\operatorname{dim}\left(Z_{i j}\right)=2$; if $E_{i} \cap Z_{i j}=\emptyset$ the plane $Z_{i j}$ projects isomorphically from $E_{i}$ onto a plane contained in $B_{i} \simeq \mathbb{P}^{1} \times \mathbb{P}^{2}$, hence $Z_{i j}$ is contained only in a unique 3 -dimensional linear space of $X_{i}$, but this is not possible by Lemma 2 iii). Therefore $E_{i} \cap Z_{i j} \neq \emptyset$ when $m=4$ too.

b) In this case $e_{i}=m-3$, hence $\operatorname{dim}\left(E_{i} \cap Z_{i j}\right) \geq m-4 \geq 0$ if $m \geq 4$.

c) In this case $e_{i}=m-2$, hence $\operatorname{dim}\left(E_{i} \cap Z_{i j}\right) \geq m-3 \geq 0$.

d) In this case $e_{i}=m-3$, hence $\operatorname{dim}\left(E_{i} \cap Z_{i j}\right) \geq m-4 \geq 0$ if $m \geq 4$.

e) In this case $e_{i}=m-2$, hence $\operatorname{dim}\left(E_{i} \cap Z_{i j}\right) \geq m-3 \geq 0$.

Corollary 6. Let $X \subset \mathbb{P}^{N}$ be an $x$-projectable Veronese variety, $\operatorname{dim}(X)=m \geq 3$. Let $X_{i}$ and $X_{j}$ be two irreducible components of $X$ which are cones of degree $d_{i}$ and $d_{j}$, having vertexes $E_{i}$ and $E_{j}$ respectively. Then:

i) if $E_{i} \cap E_{j} \neq \emptyset, d_{i}+d_{j} \leq x+1$; 
ii) if $x=3$ and $d_{i}=3, X$ contains only two components, unless $m=3$ and the vertex of $X_{i}$ (and $X_{j}$ ) is a point.

Proof. $i)$ Let $P$ be a point in $E_{i} \cap E_{j}$. By Corollary $1 \operatorname{dim}\left(\left\langle X_{j}\right\rangle \cap\left\langle X_{i}\right\rangle\right) \leq m-1$. By Lemma $4 \operatorname{dim}\left[T_{P}\left(X_{i}\right) \cup T_{P}\left(X_{j}\right)\right] \leq m+x$. Therefore, if we consider the tangent spaces at $P$, we get $\operatorname{dim}\left[T_{P}\left(X_{i}\right) \cap T_{P}\left(X_{j}\right)\right]=m+d_{i}-1+m+d_{j}-1-\operatorname{dim}\left[T_{P}\left(X_{i}\right) \cup\right.$ $\left.T_{P}\left(X_{j}\right)\right] \leq m-1$. Hence $2 m+d_{i}+d_{j}-2 \leq \operatorname{dim}\left[T_{P}\left(X_{i}\right) \cup T_{P}\left(X_{j}\right)\right]+m-1 \leq$ $2 m+x-1$.

ii) By Proposition 1 we can assume that the cone of the statement is $X_{1}$; let $E_{1}$ be its vertex. Then $\left\langle X_{1}\right\rangle \cap\left\langle X_{2}\right\rangle=X_{1} \cap X_{2} \simeq \mathbb{P}^{m-1}$ and $X_{2} \supset E_{1}$. If $X$ has another component $X_{3}$ we have $Z_{13}:=X_{3} \cap X_{1}$ is a linear space of dimension at least $m-2$ by Corollary 1 and Lemma 2 ii), moreover $Z_{13} \cap E_{1} \neq \emptyset$ by Corollary 5 , unless $m=3$ and the vertex of $X_{1}$ is a point.

Let $P$ be a point in $Z_{13} \cap E_{1}$; as we have seen $P \in X_{2}$ too. By Lemma 4 we have: $m+3 \geq \operatorname{dim}\left[T_{P}\left(X_{1}\right) \cup T_{P}\left(X_{2}\right)\right]=m+2+\operatorname{dim}\left[T_{P}\left(X_{2}\right)\right]-\operatorname{dim}\left[T_{P}\left(X_{1}\right) \cap\right.$ $\left.T_{P}\left(X_{2}\right)\right]$ hence: $m \leq \operatorname{dim}\left[T_{P}\left(X_{2}\right)\right] \leq 1+\operatorname{dim}\left[T_{P}\left(X_{1}\right) \cap T_{P}\left(X_{2}\right)\right] \leq 1+m-1=m$ and therefore: $\operatorname{dim}\left[T_{P}\left(X_{2}\right)\right]=m, \operatorname{dim}\left[T_{P}\left(X_{1}\right) \cap T_{P}\left(X_{2}\right)\right]=m-1, \operatorname{dim}\left[T_{P}\left(X_{1}\right) \cup\right.$ $\left.T_{P}\left(X_{2}\right)\right]=m+3$. This is not possible: as $P \in X_{3}$, by Lemma $3 T_{P}\left(X_{3}\right) \nsubseteq$ $\left[T_{P}\left(X_{1}\right) \cup T_{P}\left(X_{2}\right)\right]$ and we get a contradiction with Lemma 4 . Hence $X_{3}$ cannot exist unless $m=3$ and the vertex of $X_{1}$ is a point.

Corollary 7. Let $X \subset \mathbb{P}^{N}$ be a 3-projectable Veronese variety, $\operatorname{dim}(X)=m \geq 3$. Let $X_{i}$ be an irreducible component of $X$ such that $X_{i}$ is a cone of vertex $E_{i}$ and degree $d_{i}=2$. Then:

i) for any other irreducible component $X_{j}$ such that $X_{j}$ is a cone of vertex $E_{j}$ and degree $d_{j}$, if $E_{i} \cap E_{j} \neq \emptyset$, we have $d_{j}=2$;

ii) for any other irreducible component $X_{j}$ such that $X_{j}$ is a cone of vertex $E_{j}$, if $X_{j} \cap X_{i}=\left\langle X_{j}\right\rangle \cap\left\langle X_{i}\right\rangle \simeq \mathbb{P}^{m-2}$, we have $E_{i} \cap E_{j}=\emptyset$;

iii) if $m \geq 4, X$ has three components at most unless $m=4, X_{1}$ is a cone of degree 2 having a line $E_{1}$ as vertex, $X_{j} \simeq \mathbb{P}^{4}$ for any $j \geq 3$.

Proof. $i$ ) By Corollary $6 i$ ) we have: $2+d_{j} \leq 4$.

ii) By contradiction, let us assume that $E_{i} \cap E_{j} \neq \emptyset$ and let $P$ be a point in $E_{i} \cap E_{j}$. We have: $\operatorname{dim}\left[T_{P}\left(X_{i}\right)\right]=m+1$ and $\operatorname{dim}\left[T_{P}\left(X_{j}\right)\right] \geq m+1$. Let us consider the tangent spaces at $P: \operatorname{dim}\left[T_{P}\left(X_{i}\right) \cap T_{P}\left(X_{j}\right)\right]=m+1+\operatorname{dim}\left[T_{P}\left(X_{j}\right)\right]-\operatorname{dim}\left[T_{P}\left(X_{i}\right) \cup\right.$ $\left.T_{P}\left(X_{j}\right)\right] \leq m-2$. It follows: $2 m+2 \leq m+1+\operatorname{dim}\left[T_{P}\left(X_{j}\right)\right] \leq \operatorname{dim}\left[T_{P}\left(X_{i}\right) \cup\right.$ $\left.T_{P}\left(X_{j}\right)\right]+m-2$ and $m+2 \leq 1+\operatorname{dim}\left[T_{P}\left(X_{j}\right) \leq \operatorname{dim}\left[T_{P}\left(X_{i}\right) \cup T_{P}\left(X_{j}\right)\right]-2\right.$. Hence $\operatorname{dim}\left[T_{P}\left(X_{i}\right) \cup T_{P}\left(X_{j}\right)\right] \geq m+4$ and this is not possible by Lemma 4 .

iii) By Proposition 1 we can assume that the cone of the statement is $X_{1}$. Then $\left\langle X_{1}\right\rangle \cap\left\langle X_{2}\right\rangle=X_{1} \cap X_{2} \simeq \mathbb{P}^{m-1}$ and $X_{2} \supset E_{1}$. By contradiction let us assume that $X$ contains two other components $X_{3}$ and $X_{4}$ at least. By Corollary 5 we know that $Z_{13} \cap E_{1} \neq \emptyset$ and $Z_{14} \cap E_{1} \neq \emptyset$.

If $\operatorname{dim}\left(E_{1}\right)=m-2$ we have $Z_{13} \cap Z_{14} \cap E_{1} \neq \emptyset$ (as $\operatorname{dim}\left(Z_{13} \cap E_{1}\right) \geq m-3$ and $\left.\operatorname{dim}\left(Z_{14} \cap E_{1}\right) \geq m-3\right)$ so that there exists at least a point $P \in X_{1} \cap X_{2} \cap X_{3} \cap X_{4}$, but this is not possible because $\operatorname{dim}\left[T_{P}\left(X_{1}\right)\right]=m+1$ and by applying Lemma 3 we would get a contradiction with Lemma 4 .

If $\operatorname{dim}\left(E_{1}\right)=m-3$ we can argue in the same way unless $m=4$. If $m=4$ we can argue in the same way unless, for any $j \geq 3, Z_{1 j}$ are planes intersecting the line $E_{1}$ at different points $P_{1 j}$. Let us show that this is possible only if $X_{j} \simeq \mathbb{P}^{4}$ for any $j \geq 3$. Every plane $Z_{1 j}$ belongs to a unique 3 -dimensional linear space contained in 
$X_{1}$ because $Z_{1 j}$ projects from $E_{1}$ onto a line of the quadric which is the base of the cone $X_{1}$. Let $F$ be a generic 3-dimensional linear space contained in $X_{1}$. We have $F \cap Z_{1 j}=P_{1 j}$, hence $F \cap X_{j}=F \cap\left\langle X_{j}\right\rangle=P_{1 j}$. Let us consider $\left[F ; X_{j}\right]$. By Lemma 1 we have $\operatorname{dim}\left(\left[F ; X_{j}\right]\right)=8$, unless all tangent spaces at smooth points of $X_{j}$ pass through $P_{1 j}$. If $\operatorname{dim}\left(\left[F ; X_{3}\right]\right)=8$ we would have $\operatorname{dim}[\operatorname{Sec}(X)] \geq 8$, contradiction; in the other case $X_{j}$ can be a cone, having a vertex intersecting $E_{1}$, or a linear space, but if $X_{j}$ is a cone we would get a contradiction with $\left.i i\right)$ as $\operatorname{dim}\left(Z_{1 j}\right)=2$, hence $X_{j}$ is a linear space.

\section{2-Projectable Veronese VARIETiEs}

The direct generalization of Definition 1 would be the definition of 2-projectable Veronese varieties. In fact the target space for surfaces has dimension $4=\operatorname{dim}(X)+$ 2 . However the previous definition is not very interesting by the following theorem.

Theorem 4. There are no 2-projectable Veronese varieties of dimension $m \geq 3$.

Proof. Let $X \subset \mathbb{P}^{N}$ be a 2 -projectable Veronese variety of dimension $m \geq 3$. First of all let us remark that we can argue as in Lemmas 2, 3, 4 and we can prove that for any point $P \in X$ there pass at most 3 irreducible components of $X$.

Let us choose a generic linear space $L$ in $\mathbb{P}^{N}$ of codimension $m-2$. Let $S$ be $X \cap L$, then $S$ is a reducible Veronese surface according to Definition 1 . In fact $L$ cuts $\Lambda$ along a 4 -dimensional linear space and the projection of $S$ into it from $L \cap \mathcal{L}$ is an isomorphism because $\pi_{\mathcal{L}}(X)$ is isomorphic to $X$, hence $\operatorname{dim}[\operatorname{Sec}(S)] \leq 4$. Obviously $i$ ) and $i i$ ) of Definition 1 are satisfied for some $n$ by conditions $i$ ) and $i i$ ) of Definition 3. By Proposition 2.1 of $[\mathrm{E}-\mathrm{G}-\mathrm{H}-\mathrm{P}] S$ is small, hence we can give an ordering to the irreducible components $\left\{S_{j}\right\}$ of $S$ such that $\left(S_{1} \cup \ldots \cup S_{j}\right) \cap S_{j+1}=$ $\left\langle S_{1} \cup \ldots \cup S_{j}\right\rangle \cap\left\langle S_{j+1}\right\rangle$ for any $j \geq 1$, (Theorem 0.4 of [E-G-H-P]). The dimension of these linear spaces must be 1 , otherwise $X$ could not be connected in codimension 1 , and this implies that $S$ is connected in codimension 1 too. Moreover the coordinate ring of $S$ is Cohen Macaulay by Theorem 1.4 of [E-G-H-P], hence $S$ is locally Cohen Macaulay. Therefore $S$ is one of the surfaces classified by Theorem 1 .

If $S$ is a surface $\Sigma_{n}$ for some $n \geq 1$, then $X$ is the union of $n+3$ linear spaces of dimension $m$. One of them, say $X_{0}$, is cut from $L$ along $\Pi_{0}$ and the other ones, say $X_{i}$, are cut from $L$ along $\Pi_{i}$. It follows that every $X_{i}$ cut $X_{0} \simeq \mathbb{P}^{m}$ along a hyperplane, and as for any point $P \in X_{0}$ at most other two components of $X$ can pass, in $X_{0} \simeq \mathbb{P}^{m}$ three or more hyperplanes can not intersect. This is possible only if $n=2$ as $m \geq 3$ and, in this case, $X=X_{0} \cup X_{1} \cup X_{2}, \operatorname{dim}\left(X_{0} \cap X_{1}\right)=\operatorname{dim}\left(X_{0} \cap\right.$ $\left.X_{2}\right)=m-1, \operatorname{dim}\left(X_{1} \cap X_{2}\right)=m-2$. However, in this case, $\operatorname{dim}(\langle X\rangle)=m+2$, while $N$ must be $m+3$ at least.

If $S$ is the union of a smooth quadric $Q \subset \mathbb{P}^{3}$ and two planes as in Theorem 1, then $X$ must be the union of a quadric $Q^{\prime}$ and two linear spaces of dimension $m$ and they must cut $Q^{\prime}$ along a linear space of dimension $m-1$ by Theorem 3 . As in the proof of Corollary 2 the quadric $Q^{\prime}$ must be a cone over $Q$ having a vertex of dimension $m-3$, so that $X$ is a cone over $S$ having a vertex of the same dimension. For any point $P \in \operatorname{Sing}(X), \operatorname{dim}\left[T_{P}(X)\right]=\operatorname{dim}(\langle X\rangle)=m-3+\operatorname{dim}(\langle S\rangle)+1=$ $m+3=N$, so that, in this case too, $X$ is not 2-projectable because the Zariski tangent space at $P$ concides with $\mathbb{P}^{N}$. 


\section{4. $x$-PROJECTABLE VERONESE VARIETIES HAVING ONLY LINEAR COMPONENTS}

We need the following Lemma.

Lemma 5. Let $X=X_{1} \cup \ldots \cup X_{r} \subset \mathbb{P}^{N}$ be an $x$-projectable Veronese variety, such that all components of $X$ are linear spaces, $\operatorname{dim}(X)=m \geq 3$. Then, for any good ordering $X_{1}, \ldots, X_{r}$ of the components of $X$ :

i) $r \geq x+2$;

ii) for any integer $q, r \geq q \geq 2, \operatorname{dim}\left(X_{1} \cap X_{2} \cap \ldots \cap X_{q}\right) \geq m+1-q$;

iii) $X$ cannot contain three components $X_{i}, X_{j}, X_{k}$, such that $X_{i} \cap X_{j} \cap X_{k}=$ $\mathbb{P}^{m-1}$

Proof. $i$ ) We have $N=\operatorname{dim}(\langle X\rangle)=m+r-1 \geq m+x+1$. Thus implies $r \geq x+2$.

ii) Let us prove the following claim: for any integer $p, r \geq p \geq 2, \operatorname{dim}\left(X_{1} \cap \ldots \cap\right.$ $\left.X_{p-1}\right) \geq \operatorname{dim}\left(X_{1} \cap \ldots \cap X_{p}\right) \geq \operatorname{dim}\left(X_{1} \cap \ldots \cap X_{p-1}\right)-1$.

In fact, by Corollary $1 \mathrm{ii}$ ) there exists at least a component $X_{s}$, with $s<p$, such that $X_{p} \cap X_{s} \simeq \mathbb{P}^{m-1}$. Therefore $X_{1} \cap \ldots \cap X_{p}$ is the intersection of the linear space $X_{1} \cap \ldots \cap X_{p-1} \subset X_{j} \simeq \mathbb{P}^{m}$ with a hyperplane of $X_{s}$, hence the claim follows.

From the claim it follows that $\operatorname{dim}\left(X_{1} \cap \ldots \cap X_{q}\right) \geq \operatorname{dim}\left(X_{1}\right)-(q-1)=m+1-q$.

iii) By contradiction, let us assume that $X$ contains three components $X_{i}, X_{j}$, $X_{k}$, such that $X_{i} \cap X_{j} \cap X_{k}:=\Pi \simeq \mathbb{P}^{m-1}$. By Proposition 1 we can choose a good ordering among the components of $X$, such that $X_{1}=X_{i}$. We can also assume that $2 \leq j<k$.

The claim proved in $i i$ ) implies that, for any integer $q$ with $r \geq q \geq 2$, if there exists an integer $q^{\prime}$ with $2 \leq q^{\prime} \leq q$ such that $X_{1} \cap \ldots \cap X_{q^{\prime}-1}=X_{1} \cap \ldots \cap X_{q^{\prime}}$ then $\operatorname{dim}\left(X_{1} \cap \ldots \cap X_{q}\right) \geq m+2-q$.

Let us consider three cases, by recalling that $r \geq x+2$ by $i$ ) and that $m \geq x$.

a) $2 \leq j<k \leq x+2$. In this case $X_{1} \cap \ldots \cap X_{k-1}=X_{1} \cap \ldots \cap X_{k}$, hence $\operatorname{dim}\left(X_{1} \cap \ldots \cap X_{x+2}\right) \geq m+2-(x+2) \geq 0$ by $\left.i i\right)$. Then there is a point, at least, contained in $x+2$ components of $X$ and this is a contradiction with Corollary 4 .

b) $2 \leq j \leq x+1<k$. In this case $\operatorname{dim}\left(X_{1} \cap \ldots \cap X_{x+1}\right) \geq m+1-(x+1) \geq 0$ by $i$ ). Let $P$ be a point in $X_{1} \cap \ldots \cap X_{x+1}, P \in X_{k}$ too by assumptions, then there is a point, at least, contained in $x+2$ components of $X$ and this is a contradiction with Corollary 4.

c) $2<x<j<k$. In this case $\operatorname{dim}\left(X_{1} \cap \ldots \cap X_{x}\right) \geq m+1-x \geq 1$, by $\left.i i\right)$. Therefore in $X_{1}$ there is at least a point $P \in X_{1} \cap \ldots \cap X_{x} \cap \Pi$, hence there is a point, at least, contained in $x+2$ components of $X$ and this is a contradiction with Corollary 4.

Lemma 6. Let $X=X_{1} \cup X_{2} \cup \ldots \cup X_{r} \subset \mathbb{P}^{N}$ be an $x$-projectable Veronese variety, $\operatorname{dim}(X)=m \geq 3$. If all components of $X$ are linear spaces then the components of $X$ are the vertexes of a connected graph $\mathcal{A}$ which is a tree, having $X_{1}$ as root; the edges of $\mathcal{A}$ correspond to pairs $X_{i}, X_{j}$ such that $X_{i} \cap X_{j} \simeq \mathbb{P}^{m-1}$, moreover $m=x$ and no simple path is longer than $x$. If $x=m=3$ we have only the following possibilities:

$i)$ there is a distinguished component $X_{i}$ and all other components intersect $X_{i}$ along planes of $X_{i}$ in general position, $r \geq 5$;

ii) there are two distinguished components $X_{i}$ and $X_{j}$ such that $X_{i} \cap X_{j}:=$ $\Pi \simeq \mathbb{P}^{2}$ and all other components intersect $X_{i}$ along planes in general position, or intersect $X_{j}$ along planes in general position, and intersect each other along lines in general position in $\Pi ; r \geq 5$. 
Proof. Let us cut $X$ with a linear space of codimension $m-1$ in general position. Let $C$ be the linear section. $C=C_{1} \cup C_{2} \cup \ldots \cup C_{r}$ is a curve having exactly $r$ irreducible components which are lines. Note that $C_{i} \cap C_{j} \neq \emptyset$ if and only if $\operatorname{dim}\left(X_{i} \cap X_{j}\right)=m-1$. As $X$ is 1 -connected $C$ is connected and by Lemma 5 there are no points on $C$ for which there pass more than two lines, moreover $C$ is small according to the definition of [E-G-H-P]. By Proposition 2.1 of that paper there are no 3 -secant lines for $C$, no 4 -secant planes for $C, \ldots$, no $k$-secant linear spaces of dimension $k-2$ (see Theorem 2.2 of [E-G-H-P]). It follows that if we consider a graph $\mathcal{A}$ such that the vertexes of $\mathcal{A}$ are the components of $C$ and the edges of $\mathcal{A}$ correspond to the intersections among the components of $C$, then $\mathcal{A}$ is a (connected) tree, because there are no circuits in $\mathcal{A}$.

Now, if we consider the weighted graph $\mathcal{G}_{X}$ associated to $X$ as in [E-G-H-P], page 1381, in our case $\mathcal{G}_{X}$ is a complete graph by Lemma $2 i i$ ), the graph $\mathcal{A}$ is a spanning tree for $\mathcal{G}_{X}$ and $X_{1}$ gives the root of $\mathcal{A}$. Moreover the order among the components of $X$ is compatible with the natural order of vertexes in $\mathcal{A}$ (see Theorem 5.1 (b) of [E-G-H-P]).

By contradiction, let us assume that $m \geq x+1$. By Lemma $5 i$ ), we have $r \geq x+2$. By taking $q=x+2$, Lemma 5 ii $)$ implies that $\operatorname{dim}\left(X_{1} \cap X_{2} \cap \ldots \cap X_{x+2}\right) \geq$ $m-(x+2)+1 \geq 0$ and this is a contradiction with Corollary 4 ; hence $m \leq x$. As $m \geq x$ in any case, we get $m=x$.

Let us consider a fixed component $X_{k}$ in $X, k \neq 1$. There is a unique path $\gamma$ in $\mathcal{A}$ joining $X_{k}$ with the root $X_{1}$. We want to prove that $\gamma$ has no more than $x$ edges. Let us assume that $X_{1=p_{1}}, X_{p_{2}}, \ldots, X_{p_{t}}=X_{k}$ give rise to the unique path in $\mathcal{A}$ joining $X_{k}$ with $X_{1}$ and let us prove that $X_{p_{1}} \cap X_{p_{t}}=X_{p_{1}} \cap X_{p_{2}} \cap \ldots \cap X_{p_{t}}$. Obviously we have only to prove that $X_{p_{1}} \cap X_{p_{t}} \subseteq X_{p_{1}} \cap X_{p_{2}} \cap \ldots \cap X_{p_{t}}$. Let us do induction on $t$ : if $t=2$ it is true. If it is true for $2, \ldots, t-1$ we have $X_{p_{1}} \cap X_{p_{t-1}} \subseteq X_{p_{1}} \cap X_{p_{2}} \cap \ldots \cap X_{p_{t-1}}$, on the other hand $X_{p_{1}} \cap X_{p_{t}} \subseteq X_{p_{t-1}}$ by Corollary $1 \mathrm{ii}$ ) because $X_{p_{t}} \cap X_{p_{t-1}} \simeq \mathbb{P}^{m-1}$. Hence $X_{p_{1}} \cap X_{p_{t}} \subseteq X_{p_{1}} \cap X_{p_{t-1}} \subseteq$ $X_{p_{1}} \cap X_{p_{2}} \cap \ldots \cap X_{p_{t-1}} \subseteq X_{p_{1}} \cap X_{p_{2}} \cap \ldots \cap X_{p_{t}}$.

By contradiction, let us assume that $t \geq x+2$. As $\operatorname{dim}\left(X_{1} \cap X_{k}\right) \geq 0$ by Lemma 2 ii) we have $\operatorname{dim}\left(X_{p_{1}} \cap X_{p_{2}} \cap \ldots \cap X_{p_{t}}\right) \geq 0$ by the previous claim, proved by induction, but this is a contradiction with Corollary 4.

Now let us consider any two components of $X$, say $X_{j}$ and $X_{k}$, and, by contradiction, let us assume that there exists a path $\gamma$ in $\mathcal{A}$, joining $X_{k}$ with $X_{j}$, having more than $x$ edges. Let us recall that there exists a good ordering such that $X_{j}=X_{1}^{\prime}$. With respect to this new order we get another tree $\mathcal{A}^{\prime}$ for which the root is $X_{j}$, however $\mathcal{A}^{\prime}$ has the same edges of $\mathcal{A}$, so that we would have a path in $\mathcal{A}^{\prime}$, having more than $x$ edges, joining $X_{k}$ and the root of $\mathcal{A}^{\prime}$ : contradiction.

If $x=m=3$, in $\mathcal{A}$ every path passing through the root $X_{1}$ has 3 edges at most. It is easy to see that the only possibilities for $X$ are $i$ ) or $i i$ ), obviously with $r \geq 5$ since $N \geq 7$.

Now we can give the complete classification of $x$-projectble varieties $X$ when all the components of $X$ are linear spaces.

Theorem 5. Let $X=X_{1} \cup X_{2} \cup \ldots \cup X_{r} \subset \mathbb{P}^{N}$ be a variety satisfying the assumptions of Theorem $3, \operatorname{dim}(X)=m \geq 3$, such that all components of $X$ are linear spaces. Then $X$ is $x$-projectable if and only if:

i) $r \geq x+2$ 
ii) $\operatorname{dim}\left(X_{i} \cap X_{j}\right) \geq 0$ for any pair of components of $X$;

iii) the components of $X$ are the vertexes of a connected graph $\mathcal{A}$ which is a tree, having $X_{1}$ as root; the edges of $\mathcal{A}$ correspond to pairs $X_{i}, X_{j}$ such that $X_{i} \cap X_{j} \simeq$ $\mathbb{P}^{m-1}$; the natural ordering of the vertexes of $A$ is compatible with a good ordering;

$i v)$ every point $P \in X$ is contained in at most $x+1$ components of $X$.

Proof. By Lemma 2 ii), Corollary 4, Lemma 5 i) and Lemma 6 we know that the above assumptions are necessary.

As $X$ satisfies the assumptions of Theorem 3, we know that $i$ ), ii), iv) and $v$ ) of Definition 3 are satisfied (for $v$ ) recall the proof of Theorem 4). By looking at the proofs of Lemma 2 and Lemma 6 we see that the above assumptions imply that $m=x$ and that in $\mathcal{A}$ there are no paths whose lenght is longer than $x$.

Moreover assumption $i$ ) implies that $N=m+r-1 \geq m+x+1$ and $i$ i) implies that $\operatorname{dim}\left(\left[X_{i} ; X_{j}\right]\right)=\operatorname{dim}\left(X_{i} \cup X_{j}\right) \leq m+x$ for any pair of components of $X$, hence $\operatorname{dim}[\operatorname{Sec}(X)] \leq m+x$. To get iii) of Definition 3 we have only to prove that the union of all Zariski tangent spaces at any point $P \in X$ has dimension at most $m+x=2 m$. As $X$ is an union of linear spaces it suffices to prove that, for any point $P \in X$, the linear span of all linear spaces of $X$ containing $P$ has dimension at most $2 m$.

Let $P$ be a point of $X$, by Proposition 1 we can assume that $P \in X_{1}$, the root of $\mathcal{A}$. Let us assume that $P$ is contained in $s$ components of $X$ and let $X_{k}$ be one of these components. There is a unique path $\gamma_{k}, X_{1=p_{1}} \leftarrow X_{p_{2}} \leftarrow \ldots \leftarrow X_{p_{t}}=X_{k}$, joining $X_{k}$ and $X_{1}$ in $\mathcal{A}$, with $1=p_{1}<p_{2}<\ldots<p_{t}=k$ and, by the proof of Lemma 6, we know that $P \in X_{p_{1}} \cap X_{p_{2}} \cap \ldots \cap X_{p_{t}}$.

We consider the components $X_{k}$ containing $P$ such that the corresponding path $\gamma_{k}$ is of maximal lenght; let us call them $\mathrm{m}_{P}$-components. Let $\Gamma_{k}$ be the set of vertexes of $\mathcal{A}$ involved by $\gamma_{k}$. We can give a complete, not unique, ordering

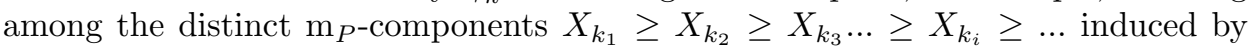
a corresponding complete, not unique, ordering on the sets $\left\{\Gamma_{k}\right\}$ defined in the following way:

$\Gamma_{k_{1}} \geq \Gamma_{k_{2}} \geq \Gamma_{k_{3}} \ldots \geq \Gamma_{k_{i}} \geq \ldots$ if and only if

$\Gamma_{k_{1}} \supseteq \Gamma_{k_{1}} \cap \Gamma_{k_{2}} \supseteq\left(\Gamma_{k_{1}} \cup \Gamma_{k_{2}}\right) \cap \Gamma_{k_{3}} \ldots \supseteq\left(\Gamma_{k_{1}} \cup \Gamma_{k_{2}} \cup \ldots \cup \Gamma_{k_{i-1}}\right) \cap \Gamma_{k_{i}} \supseteq \ldots$

For instance, in the following tree:

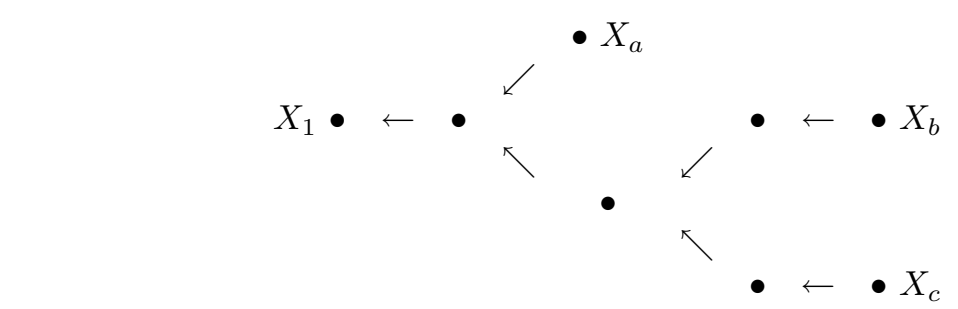

$X_{c} \geq X_{b} \geq X_{a}$ or $X_{b} \geq X_{c} \geq X_{a}$ are allowable orderings, $X_{a} \geq X_{b} \geq X_{c}$ is not allowable.

Now let us consider the first $\mathrm{m}_{P}$-component $X_{k_{1}}$ and the corresponding path $\gamma_{k_{1}}$ as above. We have $\operatorname{dim}\left(X_{p_{1}} \cup X_{p_{2}} \cup \ldots \cup X_{p_{t}}\right)=m+t-1$. Let us prove it by induction on $t \geq 2$ : if $t=2$ it is true; let us assume that it is true for $2, \ldots, t-1$, then:

$$
\begin{aligned}
& \operatorname{dim}\left(X_{p_{1}} \cup X_{p_{2}} \cup \ldots \cup X_{p_{t}}\right)=\operatorname{dim}\left[\left(X_{p_{1}} \cup X_{p_{2}} \cup \ldots \cup X_{p_{t-1}}\right) \cup X_{p_{t}}\right]= \\
& =\operatorname{dim}\left(X_{p_{1}} \cup X_{p_{2}} \cup \ldots \cup X_{p_{t-1}}\right)+\operatorname{dim}\left(X_{p_{t}}\right)-\operatorname{dim}\left[\left(X_{p_{1}} \cup X_{p_{2}} \cup \ldots \cup X_{p_{t-1}}\right) \cap X_{p_{t}}\right]=
\end{aligned}
$$


$=m+t-2+m-(m-1)=m+t-1$,

because $\left(X_{p_{1}} \cup X_{p_{2}} \cup \ldots \cup X_{p_{t-1}}\right) \cap X_{p_{t}}=X_{p_{t-1}} \cap X_{p_{t}}$ by the properties of $\mathcal{A}$ given by assumption $i v)$. In fact $\left\{X_{p_{1}}, X_{p_{2}}, \ldots, X_{p_{t-1}}\right\}$ are among $\left\{X_{1}, X_{2}, \ldots, X_{p_{t}-1}\right\}$, as the two orderings are compatible, and $\left(X_{1} \cup X_{2} \cup \ldots \cup X_{p_{t}-1}\right) \cap X_{p_{t}}=X_{p_{t-1}} \cap X_{p_{t}}$ by Corollary $1 \mathrm{ii}$ ).

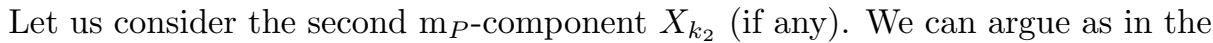
previous case: there is a unique path $\gamma_{k_{2}}, X_{1=q_{1}} \leftarrow X_{q_{2}} \leftarrow \ldots \leftarrow X_{q_{\tau}}=X_{k_{2}}$, joining $X_{k_{2}}$ and $X_{1}$ in $\mathcal{A}$, with $1=q_{1}<q_{2}<\ldots<q_{\tau}=k_{2}, P \in X_{q_{1}} \cap X_{q_{2}} \cap \ldots \cap X_{q_{\tau}}$, and $\operatorname{dim}\left(X_{q_{1}} \cup X_{q_{2}} \cup \ldots \cup X_{q_{\tau}}\right)=m+\tau-1$. Of course there are some components common to $\gamma_{k_{1}}$ and $\gamma_{k_{2}}$ (at least $X_{1}$ ): if $p_{1}=q_{1}, p_{2}=q_{2}, \ldots, p_{z}=q_{z}$ for some integer $z \geq 1$, then $\operatorname{dim}\left(X_{p_{1}} \cup X_{p_{2}} \cup \ldots \cup X_{p_{t}} \cup X_{q_{1}} \cup X_{q_{2}} \cup \ldots \cup X_{q_{\tau}}\right)=$

$=\operatorname{dim}\left(X_{p_{1}} \cup X_{p_{2}} \cup \ldots \cup X_{p_{t}}\right)+\operatorname{dim}\left(X_{q_{1}} \cup X_{q_{2}} \cup \ldots \cup X_{q_{\tau}}\right)$

$-\operatorname{dim}\left[\left(X_{p_{1}} \cup X_{p_{2}} \cup \ldots \cup X_{p_{t}}\right) \cap\left(X_{q_{1}} \cup X_{q_{2}} \cup \ldots \cup X_{q_{\tau}}\right)\right] \leq$

$\leq(m+t-1)+(m+\tau-1)-\operatorname{dim}\left(X_{q_{1}} \cup X_{q_{2}} \cup \ldots \cup X_{q_{z}}\right)=$

$=2 m+t+\tau-2-(m+z-1)=m+t+\tau-z-1$.

Note that $t+\tau-z$ is exactly the number of components of $X$ containing $P$ and involved by $\gamma_{k_{1}}$ and $\gamma_{k_{2}}$.

Now let $X_{k_{3}}$ be the third $\mathrm{m}_{P}$-component (if any). As above, there is a unique path $\gamma_{k_{3}}, X_{1=v_{1}} \leftarrow X_{v_{2}} \leftarrow \ldots \leftarrow X_{v_{\theta}}=X_{k_{3}}$, joining $X_{k_{3}}$ and $X_{1}$ in $\mathcal{A}$, with $1=v_{1}<$ $v_{2}<\ldots<v_{\theta}=k_{3}, P \in X_{v_{1}} \cap X_{v_{2}} \cap \ldots \cap X_{v_{\theta}}$, and $\operatorname{dim}\left(X_{v_{1}} \cup X_{v_{2}} \cup \ldots \cup X_{v_{\theta}}\right)=m+\theta-1$. Of course there are some components common to $\gamma_{k_{1}}, \gamma_{k_{2}}$ and $\gamma_{k_{3}}$ (at least $X_{1}$ ). Thanks to the given ordering among the $\mathrm{m}_{P}$-components, we have to consider only the case in which $p_{1}=q_{1}=v_{1}, p_{2}=q_{2}=v_{2}, \ldots, p_{w}=q_{w}=v_{w}$ for some integer $w \geq 1$. Then $\operatorname{dim}\left(X_{p_{1}} \cup \ldots \cup X_{p_{t}} \cup X_{q_{1}} \cup \ldots \cup X_{q_{\tau}} \cup X_{v_{1}} \cup \ldots \cup X_{v_{\theta}}\right)=$

$=\operatorname{dim}\left(X_{p_{1}} \cup \ldots \cup X_{p_{t}} \cup X_{q_{1}} \cup \ldots \cup X_{q_{\tau}}\right)+\operatorname{dim}\left(X_{v_{1}} \cup \ldots \cup X_{v_{\theta}}\right)$

$-\operatorname{dim}\left[\left(X_{p_{1}} \cup \ldots \cup X_{p_{t}} \cup X_{q_{1}} \cup \ldots \cup X_{q_{\tau}}\right) \cap\left(X_{v_{1}} \cup \ldots \cup X_{v_{\theta}}\right)\right] \leq$

$\leq(m+t+\tau-z-1)+(m+\theta-1)-\operatorname{dim}\left(X_{v_{1}} \cup X_{v_{2}} \cup \ldots \cup X_{v_{w}}\right)=$

$=2 m+t+\tau+\theta-z-2-(m+w-1)=m+t+\tau+\theta-z-w-1$.

Note that $t+\tau+\theta-z-w$ is exactly the number of components of $X$ containing $P$ and involved by $\gamma_{k_{1}}, \gamma_{k_{2}}$ and $\gamma_{k_{3}}$.

By iterating the same calculation for all $\mathrm{m}_{P}$-components of $X$, we have that the dimension of the linear span of all components containing $P$ is at most $m+s-1$. As $s \leq x+1$ by assumption $i v)$, it is at most $m+x=2 m$.

\section{3-Projectable Veronese Varieties with $m \geq 4$}

In this section we will prove the following theorems:

Theorem 6. Let $X \subset \mathbb{P}^{N}$ be a 3-projectable Veronese variety, $\operatorname{dim}(X)=m \geq 4$. Then $X$ cannot contain a cone of degree 3 .

Proof. By contradiction, let us assume that $X$ contains a cone $X_{i}$ of degree 3 as a component. By Corollary 5 there exists a good ordering among the components of $X$, such that $i=1$. Let $E_{1}$ be the vertex of $X_{1}$. By Corollary 6 we know that there exists only another component $X_{2}$ such that $X_{2} \cap X_{1}=\left\langle X_{2}\right\rangle \cap\left\langle X_{1}\right\rangle \simeq \mathbb{P}^{m-1}$. If $X_{2} \simeq \mathbb{P}^{m-1}$ then $\operatorname{dim}\left(\left\langle X_{1} \cup X_{2}\right\rangle\right)=(m+2)+m-(m-1)=m+3$, but we are assuming $N>m+3$, so that $X_{2}$ cannot be a linear space. By Propositions 2,3 we know that $X_{2}$ is a cone; let $E_{2}$ be its vertex. By Corollary $6 i$ ) we have: $E_{1} \cap E_{2}=\emptyset$.

Let $F_{1}$ be a generic $(m-1)$-dimensional linear space contained in $X_{1}$, different from $X_{2} \cap X_{1}$. Let $F_{2}$ be a generic $(m-1)$-dimensional linear space contained in 
$X_{2}$, different from $X_{2} \cap X_{1}$. If $m \geq 5$, by Lemma $\left.2 i i i\right)$ we know that $F_{1} \cap F_{2} \neq \emptyset$, but this implies $E_{1} \cap E_{2} \neq \emptyset$, as $F_{1} \cap\left\langle X_{2}\right\rangle=E_{1}$ and $F_{2} \cap\left\langle X_{1}\right\rangle=E_{2}$, and this is not possible. The only possibility is $m=4$.

If $m=4, X_{2} \cap X_{1}=\left\langle X_{2}\right\rangle \cap\left\langle X_{1}\right\rangle \simeq \mathbb{P}^{3}$. Let us consider $\left[F_{1} ; X_{2}\right]$. As $X$ is a 3 -projectable Veronese variety we know that $\operatorname{dim}\left(\left[F_{1} ; X_{2}\right]\right) \leq 7$. On the other hand, by Lemma $1, \operatorname{dim}\left(\left[F_{1} ; X_{2}\right]\right)=3+4-\operatorname{dim}\left[F_{1} \cap T_{P}\left(X_{2}\right)\right]$ where $P$ is a generic point of $X_{2}$, hence $\operatorname{dim}\left[F_{1} \cap T_{P}\left(X_{2}\right)\right] \geq 0$. As $F_{1} \cap\left\langle X_{2}\right\rangle=E_{1}$ we get: $E_{1} \cap T_{P}\left(X_{2}\right) \neq \emptyset$ for generic points $P \in X_{2}$. By arguing in the same way we get: $E_{2} \cap T_{Q}\left(X_{1}\right) \neq \emptyset$ for generic points $Q \in X_{1}$. It follows that $E_{1}$ and $E_{2}$ cannot be points, otherwise they would belong to the vertex of $X_{2}$ or of $X_{1}$, respectively (see [A2], page 17), and this is not possible as $E_{1} \cap E_{2}=\emptyset$. $E_{1}$ and $E_{2}$ cannot be planes as $E_{1} \cap E_{2}=\emptyset$. So that the only possibility is that $\operatorname{dim}\left(E_{1}\right)=\operatorname{dim}\left(E_{2}\right)=1$.

Let us consider the line $E_{2}$ in $X_{1}$. As $E_{2}$ is contained in $X_{2} \cap X_{1}$ and it is disjoint with $E_{1}$, it is contained in a unique 3 -dimensional linear space of $X_{1}$, which is a cone over a cubic scroll $B_{1} \subset \mathbb{P}^{4}$ because $E_{2}$ projects from $E_{1}$ onto a line $l$ of $B_{1}$. Let us call $\pi$ the projection. As $E_{2} \cap T_{Q}\left(X_{1}\right) \neq \emptyset$ for generic points $Q \in X_{1}$ we get $l \cap T_{\pi(Q)}\left(B_{1}\right) \neq \emptyset$ for generic points $Q \in X_{1}$, hence $l \cap T_{b}\left(B_{1}\right) \neq \emptyset$ for generic points $b \in B_{1}$ and this is not possible.

Theorem 7. Let $X \subset \mathbb{P}^{N}$ be a 3-projectable Veronese variety, $\operatorname{dim}(X)=m \geq 4$. If $X$ contains a cone $X_{i}$ of degree 2 then $m=4$ and:

1) if $X$ has at most 3 components, $X=X_{1} \cup X_{2} \cup X_{3}, X_{1} \cap X_{2} \cap X_{3} \simeq \mathbb{P}^{3}$, $X_{1}$ and $X_{2}$ are cones with a line as vertex, $X_{3}$ is a cone of the same type and the three vertexes are disjoint, or $X_{3} \simeq \mathbb{P}^{4}$; in the first case $X \subset \mathbb{P}^{9}$ in the second case $X \subset \mathbb{P}^{8}$

2) if $X$ has more than 3 components, $X=X_{1} \cup X_{2} \cup X_{3} \cup X_{4}, X_{1}$ is a cone with a line as vertex, $X_{i}$ are linear spaces for $i \geq 2, X_{1} \cap X_{2} \simeq \mathbb{P}^{3}, X_{1} \cap X_{2} \cap X_{3} \simeq \mathbb{P}^{2}$, $X_{1} \cap X_{2} \cap X_{3} \cap X_{4} \simeq \mathbb{P}^{1}$, (disjoint with the vertex of $X_{1}$ ), $X \subset \mathbb{P}^{8}$.

Proof. By Proposition 1 there exists a good ordering among the components of $X$, such that $i=1$. Let $E_{1}$ be the vertex of $X_{1}$. If $X$ has only two components $X_{1}$ and $X_{2}$ we know that $X_{2} \cap X_{1}=\left\langle X_{2}\right\rangle \cap\left\langle X_{1}\right\rangle \simeq \mathbb{P}^{m-1}$, and, in any case, $\operatorname{dim}\left(\left\langle X_{2} \cup X_{1}\right\rangle\right) \leq m+3$, hence this possibility does not occur as $N>m+3$ and $X$ has 3 components at least. By Proposition 3 and Theorem $6, X_{i}$ can be only a quadric cone or a linear space for any $i \geq 2$. By Corollary 7 iii) we have only two possibilities.

1) Let us assume that $X$ has exactly 3 components. We have to consider the following four cases:

a) $X_{2}$ and $X_{3}$ are quadric cones;

b) $X_{2}$ is a quadric cone and $X_{3}$ is a linear space;

c) $X_{2}$ and $X_{3}$ are linear spaces;

d) $X_{2}$ is a linear space and $X_{3}$ is a quadric cone.

a) Let $E_{2}$ be the vertex of $X_{2}$. Let $F_{1}$ be a generic $(m-1)$-dimensional linear space contained in $X_{1}$, different from $X_{2} \cap X_{1}$. Let $F_{2}$ be a generic $(m-1)$-dimensional linear space contained in $X_{2}$, different from $X_{2} \cap X_{1}$.

If $m \geq 5$, by Lemma 2 iii) we know that $F_{1} \cap F_{2} \neq \emptyset$, but this implies $E_{1} \cap E_{2} \neq \emptyset$, as $F_{1} \cap\left\langle X_{2}\right\rangle=E_{1}$ and $F_{2} \cap\left\langle X_{1}\right\rangle=E_{2}$. Let us consider $X_{3}$. We know that $X_{3} \cap\left(X_{1} \cup X_{2}\right) \simeq \mathbb{P}^{m-1}$, hence $X_{3} \cap X_{1} \simeq \mathbb{P}^{m-1}$ or $X_{3} \cap X_{2} \simeq \mathbb{P}^{m-1}$. In the first case $E_{1} \subset X_{3}$ and $E_{1} \cap E_{2} \cap X_{3} \neq \emptyset$. In the second case $E_{2} \subset X_{3}$ and $E_{1} \cap E_{2} \cap X_{3} \neq \emptyset$. So 
that there exists at least a point $P \in E_{1} \cap E_{2} \cap X_{3}$, but this is a contradiction with Lemmas 3 and 4 because $\operatorname{dim}\left[T_{P}\left(X_{1}\right) \cup T_{P}\left(X_{2}\right)\right]=(m+1)+(m+1)-(m-1)=m+3$.

If $m=4$ the above argument shows that $E_{1} \cap E_{2}=\emptyset$, hence $\operatorname{dim}\left(E_{1}\right)=$ $\operatorname{dim}\left(E_{2}\right)=1$ as $X_{2} \cap X_{1} \simeq \mathbb{P}^{3}$ (recall Proposition 2). By Lemma $2 i i$ ) we have to consider 3 subcases:

- $X_{3} \cap X_{1} \simeq \mathbb{P}^{3}$, different from $X_{2} \cap X_{1}$, and $X_{3} \cap X_{2} \simeq \mathbb{P}^{2}$ with $\left(X_{3} \cap X_{2}\right) \subset$ $\left(X_{3} \cap X_{1}\right)$. Hence $\left(X_{3} \cap X_{2}\right) \subset\left(X_{2} \cap X_{1}\right)$ and $\left(X_{3} \cap X_{2}\right) \subset\left[\left(X_{3} \cap X_{1}\right) \cap\left(X_{2} \cap X_{1}\right)\right]=E_{1}$ but this is not possible as $X_{3} \cap X_{2} \simeq \mathbb{P}^{2}$.

- $X_{3} \cap X_{2} \simeq \mathbb{P}^{3}$, different from $X_{2} \cap X_{1}$, and $X_{3} \cap X_{1} \simeq \mathbb{P}^{2}$ with $\left(X_{3} \cap X_{1}\right) \subset$ $\left(X_{3} \cap X_{2}\right)$. We can argue as before and this subcase is not possible.

- $X_{3} \cap X_{2}=X_{2} \cap X_{1} \simeq \mathbb{P}^{3}$, hence $X_{1} \cap X_{2} \cap X_{3} \simeq \mathbb{P}^{3} . X_{3}$ must be a cone having a line $E_{3}$ as vertex, because $E_{1} \cap E_{3}=\emptyset$ and $E_{2} \cap E_{3}=\emptyset$ by the above argument, and this subcase is possible.

b) We can argue as in case a), the only difference is that here $X_{3} \simeq \mathbb{P}^{4}$.

c) In this case $\operatorname{dim}\left(\left\langle X_{2} \cup X_{1}\right\rangle\right)=m+2$ and $\operatorname{dim}\left(\left\langle X_{3} \cup X_{2} \cup X_{1}\right\rangle\right)=\operatorname{dim}\left(\left\langle X_{3}\right\rangle\right)+$ $(m+2)-\operatorname{dim}\left(\left\langle X_{3}\right\rangle \cap\left\langle X_{2} \cup X_{1}\right\rangle\right)=(m+1)+(m+2)-(m-1)=m+3$, hence this possibility does not occur as $N>m+3$.

d) If $X_{3} \cap X_{1} \simeq \mathbb{P}^{m-1}$ and $X_{3} \cap X_{2} \simeq \mathbb{P}^{m-2}$ or $X_{3} \cap X_{2}=X_{3} \cap X_{1}$ we can consider a different good ordering among the components of $X$, according to Theorem 3 : $X_{1}, X_{3}, X_{2}$ and we can get case b). Hence, by Lemma 2 ii), we can assume: $X_{3} \cap$ $X_{2} \simeq \mathbb{P}^{m-1}$ and $X_{3} \cap X_{1} \simeq \mathbb{P}^{m-2}$ with $\left(X_{3} \cap X_{1}\right) \subset\left(X_{3} \cap X_{2}\right)$. Let $E_{3}$ be the vertex of $X_{3}$, by Corollary $\left.7 \mathrm{ii}\right)$ we have: $E_{1} \cap E_{3}=\emptyset$. Now, $\left(E_{1} \cap X_{3} \cap X_{1}\right) \subset$ $\left(X_{3} \cap X_{1}\right) \simeq \mathbb{P}^{m-2}$ and $E_{1} \cap X_{3} \cap X_{1}$ has dimension $\operatorname{dim}\left(E_{1}\right)-1=m-\varepsilon_{1}-1$ because $E_{1} \subset X_{2} \cap X_{1}$ and $X_{3} \cap X_{1}$ is a divisor in $X_{2} \cap X_{1}$; moreover $\left(E_{3} \cap X_{3} \cap X_{1}\right) \subset$ $\left(X_{3} \cap X_{1}\right) \simeq \mathbb{P}^{m-2}$ and $E_{3} \cap X_{3} \cap X_{1}$ has dimension $\operatorname{dim}\left(E_{3}\right)-1=m-\varepsilon_{3}-1$ because $E_{3} \subset X_{2} \cap X_{3}$ and $X_{3} \cap X_{1}$ is a divisor in $X_{2} \cap X_{3}$. As $E_{1} \cap E_{3}=\emptyset$ it must be: $m-\varepsilon_{1}-1+m-\varepsilon_{3}-1<m-2$, i.e. $m<\varepsilon_{1}+\varepsilon_{3} \leq 6$, as $\varepsilon_{i} \in\{2,3\}$. We have to consider 3 subcases:

- $m=5, \operatorname{dim}\left(E_{1}\right)=\operatorname{dim}\left(E_{3}\right)=2$. It must be $\operatorname{dim}\left(\left[X_{1} ; X_{3}\right]\right) \leq m+3=8$, hence, by Lemma 1 , it must be $\operatorname{dim}\left(\left\langle T_{P}\left(X_{1}\right) \cup T_{Q}\left(X_{3}\right)\right\rangle\right) \leq 8$ for generic points $P \in X_{1}$ and $Q \in X_{3}$. We claim that this implies that there is a fixed plane $H \subset$ $X_{3} \cap X_{1}=\left\langle X_{1}\right\rangle \cap\left\langle X_{3}\right\rangle \simeq \mathbb{P}^{3}$ such that $H \subset T_{P}\left(X_{1}\right)$ and $H \subset T_{Q}\left(X_{3}\right)$. In fact $\operatorname{dim}\left[T_{P}\left(X_{1}\right) \cap T_{Q}\left(X_{3}\right)\right] \geq 2$ and $T_{P}\left(X_{1}\right) \cap T_{Q}\left(X_{3}\right) \subseteq K:=\left\langle X_{1}\right\rangle \cap\left\langle X_{3}\right\rangle$. If $T_{P}\left(X_{1}\right)=$ $K$ for any generic pont $P \in X_{1}, X_{1}$ would be a cone with a vertex containing $K$ and this is not possible, hence $\operatorname{dim}\left[T_{P}\left(X_{1}\right) \cap K\right]=2$, analogously $\operatorname{dim}\left[T_{Q}\left(X_{3}\right) \cap K\right]=2$ for any generic pont $Q \in X_{3}$; hence $\operatorname{dim}\left[T_{P}\left(X_{1}\right) \cap T_{Q}\left(X_{3}\right)\right]=2$ for generic points $P \in X_{1}$ and $Q \in X_{3}$. Let us fix a generic point $\bar{P} \in X_{1}$ and let $\bar{H}$ be the plane $T_{\bar{P}}\left(X_{1}\right) \cap K$. As $\operatorname{dim}\left[T_{\bar{P}}\left(X_{1}\right) \cap T_{Q}\left(X_{3}\right)\right]=2$ for any generic point $Q \in X_{3}$ and $T_{\bar{P}}\left(X_{1}\right) \cap T_{Q}\left(X_{3}\right) \subseteq K$, we have $T_{\bar{P}}\left(X_{1}\right) \cap T_{Q}\left(X_{3}\right)=T_{\bar{P}}\left(X_{1}\right) \cap T_{Q}\left(X_{3}\right) \cap K=$ $\bar{H} \cap T_{Q}\left(X_{3}\right)$, hence $\bar{H} \subseteq T_{Q}\left(X_{3}\right)$ and $\bar{H} \subseteq T_{Q}\left(X_{3}\right) \cap K$. As $\operatorname{dim}\left[T_{Q}\left(X_{3}\right) \cap K\right]=2$, it follows that $\bar{H}=T_{Q}\left(X_{3}\right) \cap K$ for any generic point $Q \in X_{3}$. By changing the role of $X_{1}$ and $X_{3}$ we get the claim.

However this is not possible: let $\pi: X_{1} \rightarrow B_{1}$ be the natural projection of $X_{1}$ onto the base $B_{1}$ of the cone, $B_{1}$ is a smooth quadric in $\mathbb{P}^{3} ; \pi(H)$ is a point or a line in $B_{1}$ and the tangent space $T_{\pi(P)}\left(B_{1}\right)$ would contain $\pi(H)$ for any generic point $P \in X_{1}$ : contradiction.

- $m=4, \operatorname{dim}\left(E_{1}\right)=\operatorname{dim}\left(E_{3}\right)=1$. It must be $\operatorname{dim}\left(\left[X_{1} ; X_{3}\right]\right) \leq m+3=7$, hence, by Lemma 1 , it must be $\operatorname{dim}\left(\left\langle T_{P}\left(X_{1}\right) \cup T_{Q}\left(X_{3}\right)\right\rangle\right) \leq 7$ for generic points $P \in X_{1}$ 
and $Q \in X_{3}$. As above, this implies that there is a fixed line $L \subset X_{3} \cap X_{1}=\left\langle X_{1}\right\rangle$ $\cap\left\langle X_{3}\right\rangle \simeq \mathbb{P}^{2}$ such that $L \subset T_{P}\left(X_{1}\right)$ and $L \subset T_{Q}\left(X_{3}\right)$. But this is not possible: let $\pi: X_{1} \rightarrow B_{1}$ be the projection of $X_{1}$ onto the base $B_{1}$ of the cone, $B_{1}$ is a smooth quadric in $\mathbb{P}^{3} ; \pi(L)$ is a point or a line in $B_{1}$ and the tangent space $T_{\pi(P)}\left(B_{1}\right)$ would contain $\pi(L)$ for any generic point $P \in X_{1}$ : contradiction.

- $m=4, \operatorname{dim}\left(E_{1}\right)=1, \operatorname{dim}\left(E_{3}\right)=2$ (the case $\operatorname{dim}\left(E_{1}\right)=2, \operatorname{dim}\left(E_{3}\right)=1$ is analogous). It must be $\operatorname{dim}\left(\left[X_{1} ; X_{3}\right]\right) \leq m+3=7$, hence, by Lemma 1 , it must be $\operatorname{dim}\left(\left\langle T_{P}\left(X_{1}\right) \cup T_{Q}\left(X_{3}\right)\right\rangle\right) \leq 7$ for generic points $P \in X_{1}$ and $Q \in X_{3}$. As above, this implies that there is a fixed line $L \subset X_{3} \cap X_{1}=\left\langle X_{1}\right\rangle \cap\left\langle X_{3}\right\rangle \simeq \mathbb{P}^{2}$ such that $L \subset T_{P}\left(X_{1}\right)$ and $L \subset T_{Q}\left(X_{3}\right)$. But this is not possible: let $\pi: X_{1} \rightarrow B_{1}$ be the projection of $X_{1}$ onto the base $B_{1}$ of the cone, $B_{1}$ is a smooth quadric in $\mathbb{P}^{3} ; \pi(L)$ is a point or a line in $B_{1}$ and the tangent space $T_{\pi(P)}\left(B_{1}\right)$ would contain $\pi(L)$ for any generic point $P \in X_{1}$ : contradiction.

2) Now let us assume that $X$ has more than 3 components. By Corollary 7 we know that $X_{j}$ is a linear space for any $j \geq 3$ and by the above proof we know that $X_{2}$ can be only a quadric cone, having a line as vertex, or a linear space. In the first case, by the above proof, $X_{1} \cap X_{2} \cap X_{3}$ is a 3-dimensional linear space containing the two disjoint vertex of $X_{1}$ and $X_{2}$, so that $X_{4}$ would cut another 3-dimensional linear space on $X_{3}$, or on $X_{2}$, or on $X_{1}$, by Corollary $1 \mathrm{ii}$ ). Therefore $X_{4}$ would cut and at least a point on the two vertexes, but this is not possible by Lemma 4 . Therefore $X_{j}$ is a linear space for any $j \geq 2$.

Let $F$ be $X_{1} \cap X_{2} \simeq \mathbb{P}^{3}$, obviously $F \supset E_{1}$, the vertex of $X_{1} . X_{3}$ cuts a 3dimensional linear space on $X_{1}$, or on $X_{2}$. If $X_{3} \cap X_{1} \simeq \mathbb{P}^{3}$ then $E_{1} \subset X_{3} \cap X_{1}$, hence $E_{1} \subset X_{3} \cap X_{2} \cap X_{1}$ and $X_{4}$ would cut at least a point on $E_{1}$ : this is not possible by Lemma 4 . Therefore $X_{3} \cap X_{2} \simeq \mathbb{P}^{3}, X_{3} \cap X_{1}:=H \simeq \mathbb{P}^{2}$ (recall that $\operatorname{dim}\left(X_{3} \cap X_{1}\right) \geq 2$ by Lemma $\left.\left.2 i i\right)\right), H \subset F$ and $H=X_{3} \cap X_{2} \cap X_{1}$. Let us consider $E_{1} \cap H$; if $E_{1} \subset H$ then $X_{4}$ would cut a point on $E_{1}$, at least, and this is not possible by Lemma 4 , hence $E_{1} \cap H$ is a point $P$. By Lemma 4 no other component of $X$ passes through $P$, hence $X_{4} \cap X_{1}$ cannot be a 3 -dimensional linear space, hence $X_{4} \cap X_{2} \simeq \mathbb{P}^{3}$ or $X_{4} \cap X_{3} \simeq \mathbb{P}^{3}$, in any case $X_{4} \cap H=X_{4} \cap X_{3} \cap X_{2} \cap X_{1}$ is a line $L$, not passing through $P$, and, by Lemma 4 , no other component of $X$ intersects $L$. Now it is easy to see that $X$ cannot contain other components: $X_{5}$ would cut a point on $L$, at least, or it would pass through $P$. Both cases are not possible by Lemma 4.

\section{3-Projectable Veronese VARIEties With $m=3$}

A direct computation gives the following lemma.

Lemma 7. Let $V=\mathbb{P}(\mathcal{E})$ be a smooth rational scroll over $\mathbb{P}^{1}$ such that $\mathcal{E}=$ $\mathcal{O}_{\mathbb{P}^{1}}(2) \oplus \mathcal{O}_{\mathbb{P}^{1}}(1) \oplus \mathcal{O}_{\mathbb{P}^{1}}(1)$ or $\mathcal{E}=\mathcal{O}_{\mathbb{P}^{1}}(1) \oplus \mathcal{O}_{\mathbb{P}^{1}}(1) \oplus \mathcal{O}_{\mathbb{P}^{1}}(1)$ or $\mathcal{E}=\mathcal{O}_{\mathbb{P}^{1}}(2) \oplus \mathcal{O}_{\mathbb{P}^{1}}(1)$. Let $F$ be a fixed fibre of anyone of the above scrolls and let $P$ be a generic point of $V$. Then:

- in the first case and in the third case $T_{p}(V) \cap F=\emptyset$;

- in the second case $T_{p}(V) \cap F$ is a point and if $P$ belongs to another fibre $F^{\prime}$, the map $F^{\prime} \rightarrow F$, given by $P \rightarrow T_{p}(V) \cap F$, is an isomorphism.

Now we consider 3-projectable Veronese varieties containing scrolls and cones.

Proposition 4. Let $X \subset \mathbb{P}^{N}$ be a 3-projectable Veronese variety, $\operatorname{dim}(X)=3$. Let us assume that $X$ contains a smooth scroll $X_{i}$. Then: $X=X_{1} \cup X_{2} \cup X_{3} \subset \mathbb{P}^{7}$, 
or $X=X_{1} \cup X_{2} \cup X_{3} \cup X_{4} \subset \mathbb{P}^{8}, X_{1} \simeq \mathbb{P}^{1} \times \mathbb{P}^{2}, X_{2} \simeq X_{3} \simeq X_{4} \simeq \mathbb{P}^{3}$, and all components intersect along a fixed 2-dimensional fibre of the scroll $X_{1}$.

Proof. We can always assume that $X_{1}$ is a scroll by Proposition 1 . Then $X_{1} \cap$ $X_{2}=\left\langle X_{1}\right\rangle \cap\left\langle X_{2}\right\rangle \simeq \mathbb{P}^{2}$ is a fibre $F$ of the scroll. It must be $\operatorname{dim}\left(\left[X_{1} ; X_{2}\right]\right) \leq 6$, hence $T_{p}\left(X_{1}\right) \cap T_{Q}\left(X_{2}\right) \neq \emptyset$ by Lemma 1 for generic points $P \in X_{1}$ and $Q \in X_{2}$ and $T_{p}\left(X_{1}\right) \cap T_{Q}\left(X_{2}\right) \subseteq F$. If $X_{1}=\mathbb{P}\left(\mathcal{O}_{\mathbb{P}^{1}}(2) \oplus \mathcal{O}_{\mathbb{P}^{1}}(1) \oplus \mathcal{O}_{\mathbb{P}^{1}}(1)\right)$, by Lemma 7 , $T_{p}\left(X_{1}\right) \cap F=\emptyset$ hence $X$ cannot contain such a scroll. If $X_{1}=\mathbb{P}\left(\mathcal{O}_{\mathbb{P}^{1}}(1) \oplus \mathcal{O}_{\mathbb{P}^{1}}(1) \oplus\right.$ $\left.\mathcal{O}_{\mathbb{P}^{1}}(1)\right) \simeq \mathbb{P}^{1} \times \mathbb{P}^{2}$ and $T_{Q}\left(X_{2}\right) \cap F \neq F$ then for generic points $P \in X_{1}$ we have $T_{p}\left(X_{1}\right) \cap T_{Q}\left(X_{2}\right)=\emptyset$ by Lemma 7 . Hence $T_{Q}\left(X_{2}\right) \cap F=F, X_{2} \simeq \mathbb{P}^{3}$ and $\operatorname{dim}\left(\left\langle X_{1} \cup X_{2}\right\rangle\right)=6$. As $N \geq 7$ there exist other components in $X$. Let us consider $X_{3}$. By arguing as before we have $X_{3} \simeq \mathbb{P}^{3}$ too and $X_{3} \cap X_{1}$ is a fibre of the scroll. Since: $X_{3} \cap X_{2} \neq \emptyset, X_{3} \cap X_{2} \subseteq X_{3} \cap X_{1}$ by Corollary $1 \mathrm{ii}$ ) and the fibres of $X_{1}$ are disjoint, we have $X_{3} \cap X_{1}=F$. The same is true for $X_{4}$ if there is another component. $X$ cannot contain other components by Corollary 4.

We have no other cases to consider thanks to Proposition 3.

From now on we can assume that $X$ does not contain scrolls as components.

Proposition 5. Let $X \subset \mathbb{P}^{N}$ be a 3 -projectable Veronese variety, $\operatorname{dim}(X)=3$. Let us assume that $X$ contains a cone $X_{i}$ of degree 3 . Then $X_{i}=X_{1}, X=X_{1} \cup X_{2} \subset$ $\mathbb{P}^{7}, X_{1}$ has a line $E_{1}$ as vertex, $X_{2}$ is a cone of degree 2 having a point $E_{2}$ as vertex and $X_{1} \cap X_{2}$ is a plane containing $E_{1}$ and $E_{2}$ with $E_{1} \cap E_{2}=\emptyset$.

Proof. We can always assume that $X_{1}$ a cone of degree 3 by Proposition 1 . Then $X_{1} \cap X_{2}=\left\langle X_{1}\right\rangle \cap\left\langle X_{2}\right\rangle \simeq \mathbb{P}^{2}$ is a linear subspace $F$ of maximal dimension for the cone. It must be $\operatorname{dim}\left(\left[X_{1} ; X_{2}\right]\right) \leq 6$, hence $T_{p}\left(X_{1}\right) \cap T_{Q}\left(X_{2}\right) \neq \emptyset$ by Lemma 1 for generic points $P \in X_{1}$ and $Q \in X_{2}$ and $T_{p}\left(X_{1}\right) \cap T_{Q}\left(X_{2}\right) \subseteq F$.

If $X_{1}$ has a point $E_{1}$ as vertex and a base $B_{1} \simeq \mathbb{P}\left(\mathcal{O}_{\mathbb{P}^{1}}(2) \oplus \mathcal{O}_{\mathbb{P}^{1}}(1)\right)$ then $T_{p}\left(X_{1}\right) \cap$ $F=E_{1}$, for generic points $P \in X_{1}$, otherwise, by looking at the projection from the vertex of the cone onto $B_{1}$, we would get a contradiction with Lemma 7 . Hence $E_{1} \subset T_{Q}\left(X_{2}\right)$ and $X_{2} \simeq \mathbb{P}^{3}$ or $X_{2}$ is a cone with vertex containing $E_{1}$, but this is not possible by Corollary $6 i$ ). Therefore $X_{2} \simeq \mathbb{P}^{3}$. By arguing in this way we can conclude that all components $X_{i}$ of $X$ with $i \geq 2$ are isomorphic to $\mathbb{P}^{3}$ and they pass through $E_{1}$ (also in case $\operatorname{dim}\left(X_{i} \cap X_{1}\right)=1$ ), but this is not possible by considering $\mathbb{T}_{E_{1}}$ and Lemma 4 . The only possibility is $X=X_{1} \cup X_{2}$, but in this case $\operatorname{dim}\left(\left\langle X_{1} \cup X_{2}\right\rangle\right)=6$. As $N \geq 7, X$ cannot contain such a cone.

If $X_{1}$ has a line $E_{1}$ as vertex and a base $B_{1}$ which is a rational space cubic then $T_{p}\left(X_{1}\right) \cap F=E_{1}$, for generic points $P \in X_{1}$. Hence $E_{1} \cap T_{Q}\left(X_{2}\right) \neq \emptyset$ for generic points $Q \in X_{2}$. Let us examine the possibilities for $X_{2}$.

- If $X_{2}$ is a cone of degree 3 , or of degree 2, having a line (on $F$ ) as vertex we would get $E_{1} \cap E_{2} \neq \emptyset$, but this is not possible by Corollary $6 i$ ).

- If $X_{2}$ is a cone of degree 3 having a point as vertex we can reorder the components of $X$ changing the role of $X_{1}$ and $X_{2}$; we can argue as above and we can exclude this case.

- If $X_{2} \simeq \mathbb{P}^{3}, \operatorname{dim}\left(\left\langle X_{1} \cup X_{2}\right\rangle\right)=6$, then it must exist $X_{3}$; however $X_{3}$ would cut a plane on $X_{1}$ or on $X_{2}$ and it would intersect $E_{1}$ at least at a point $P$, but this is not possible by considering $\mathbb{T}_{P}$ and Lemma 4 .

- If $X_{2}$ is a cone of degree 2 having a point as vertex, for any generic point $Q \in X_{2}$ we have $T_{Q}\left(X_{2}\right) \cap F$ is a line passing through $E_{2}$ so that the condition $E_{1} \cap T_{Q}\left(X_{2}\right) \neq \emptyset$ is fullfilled. Of course it must be $E_{1} \cap E_{2}=\emptyset$ otherwise we would 
have a contradiction with Lemma 4. $X$ cannot contain another component $X_{3}: X_{3}$ would cut a plane or a line on $X_{1}$, hence there would be a point $P \in X_{3} \cap E_{1}$ and this is not possible by considering $\mathbb{T}_{P}$ and Lemma 4 .

For the sequel we need the following Lemma.

Lemma 8. Let $X \subset \mathbb{P}^{N}$ be a 3-projectable Veronese variety, $\operatorname{dim}(X)=3$. Let us assume that $X$ contains two cones $X_{i}$ and $X_{j}$ of degree 2 , then $X_{i} \cap X_{j}$ is a plane.

Proof. By Lemma 2 ii) we know that $X_{i} \cap X_{j}=\left\langle X_{i}\right\rangle \cap\left\langle X_{j}\right\rangle$ is a plane or a line. By contradiction, let us assume that $X_{i} \cap X_{j}$ is a line $L$ and let $\pi$ be the isomorphic projection of $X$ onto $\mathbb{P}^{6}$. In this case $\pi\left(\left\langle X_{i}\right\rangle\right) \simeq\left\langle X_{i}\right\rangle \simeq\left\langle X_{j}\right\rangle \simeq \pi\left(\left\langle X_{j}\right\rangle\right) \simeq \mathbb{P}^{4}$ and $\pi\left(\left\langle X_{i}\right\rangle\right) \cap \pi\left(\left\langle X_{j}\right\rangle\right)$ is a plane $\Pi$, moreover there are two planes $\Pi_{i} \subset\left\langle X_{i}\right\rangle$ and $\Pi_{j} \subset\left\langle X_{j}\right\rangle$ such that $L=\Pi_{i} \cap \Pi_{j}$ and $\Pi=\pi\left(\Pi_{i}\right)=\pi\left(\Pi_{j}\right)$. Let $C_{i}^{\prime} \supset L$ and $C_{j}^{\prime} \supset L$ be the two plane conics $\Pi_{i} \cap X_{i}$ and $\Pi_{j} \cap X_{j}$. As $\pi$ is an isomorphism, $C_{i}=\pi\left(C_{i}^{\prime}\right)$ and $C_{j}=\pi\left(C_{j}^{\prime}\right)$ are two plane conics in $\Pi$ and $L=X_{i} \cap X_{j} \simeq \pi\left(X_{i} \cap X_{j}\right) \simeq$ $\pi\left(X_{i}\right) \cap \pi\left(X_{j}\right)=C_{i} \cap C_{j}$ : contradiction, as the intersection of two plane conics cannot be isomorphic to a (reduced) line.

Proposition 6. Let $X \subset \mathbb{P}^{N}$ be a 3-projectable Veronese variety, $\operatorname{dim}(X)=3$. Let us assume that $X$ does not contain cones of degree 3 and that $X$ contains a cone $X_{i}$ of degree 2 having a line $E_{i}$ as vertex. Then:

i) $X=X_{1} \cup X_{2} \cup X_{3} \subset \mathbb{P}^{8} ; X_{1}$ is a quadric cone having a line $E_{1}$ as vertex, $X_{2}$ and $X_{3}$ are quadric cones having points $E_{2}$ and $E_{3}$ as vertexes; $X_{1} \cap X_{2} \cap X_{3}$ is a plane $F$, the vertexes are disjoint;

ii) $X=X_{1} \cup X_{2} \cup X_{3} \subset \mathbb{P}^{7} ; X_{1}$ is a quadric cone having a line $E_{1}$ as vertex, $X_{2}$ is a quadric cone having a point $E_{2}$ as vertex, $X_{3} \simeq \mathbb{P}^{3} ; X_{1} \cap X_{2} \cap X_{3}$ is a plane $F$, the vertexes are disjoint (the role of $X_{2}$ and $X_{3}$ can be exchanged);

iii) $X=X_{1} \cup X_{2} \cup X_{3} \subset \mathbb{P}^{7} ; X_{1}$ is a quadric cone having a line $E_{1}$ as vertex, $X_{2}$ is a quadric cone having a point $E_{2}$ as vertex, $X_{3} \simeq \mathbb{P}^{3} ; X_{1} \cap X_{2}$ is a plane $F$, $X_{3} \cap X_{1}$ is another plane $F^{\prime}, X_{3} \cap X_{2}=E_{1} \subset F$, the vertexes are disjoint (the role of $X_{2}$ and $X_{3}$ can be exchanged);

iv) $X=X_{1} \cup X_{2} \cup \ldots \cup X_{r} \subset \mathbb{P}^{r+3}, r \geq 4 ; X_{1}$ is a quadric cone having a line $E_{1}$ as vertex, $X_{i} \simeq \mathbb{P}^{3}$ for $i \geq 2 ; X_{1} \cap X_{2}$ is a plane $F ; X_{i} \cap X_{2}$ are planes in generic position in $X_{2}$ intersecting lines $L_{i} \subset F$ in generic position for $i \geq 3$.

Proof. As usual we can assume that $X_{1}$ is a quadric cone having a line $E_{1}$ as vertex by Proposition 1. We have to consider three cases.

Case 1: $X_{2}$ is a quadric cone having a line $E_{2}$ as vertex, intersecting $X_{1}$ along a plane $F$. Let $P$ be a point in $E_{1} \cap E_{2} \neq \emptyset$. If $X$ contains a component $X_{3}$ then $X_{3}$ cuts a plane on $X_{2}$ or on $X_{1}$ (or on both), in any case $X_{3}$ passes through $P$, but this is not possible by Lemma 4 . Moreover $X$ cannot be $X_{1} \cup X_{2}$ because $N \geq 7$.

Case 2: $X_{2}$ is a quadric cone having a point $E_{2}$ as vertex, intersecting $X_{1}$ along a plane $F$. If $E_{2} \in E_{1} \subset F$ we can argue as in Case 1 and we have no possibilities, so we can assume $E_{2} \notin E_{1}$. $X$ cannot be $X_{1} \cup X_{2}$ because $N \geq 7$. Let us consider $X_{3}$.

2a) $X_{3}$ is a quadric cone. Hence it cuts a plane on $X_{1}$ and $X_{2}$ by Lemma 8 and the two planes coincide with $F$ by Corollary $1 \mathrm{ii}$ ). By Lemma 4 the vertex $E_{3}$ of $X_{3}$ does not intersect $E_{1}$ or $E_{2}$, hence $E_{3}$ is a point in $F$ in generic position with respect $E_{1}$ and $E_{2} . X$ cannot contain $X_{4}$ because $X_{4}$ would cut a plane on some 
$X_{i}, i \leq 3$, hence it would pass through some vertex $E_{i}$ but this is not possible by Lemma 4. We have $i$ ).

2b) $X_{3} \simeq \mathbb{P}^{3}, X_{3} \cap X_{2}$ is a plane $F^{\prime} \neq F, X_{3} \cap X_{1}$ is a line $L \subset F^{\prime}$. As $F^{\prime} \cap X_{1}=E_{2}$, the assumptions imply that $F^{\prime}=F$, so that this subcase is not possible.

2c) $X_{3} \simeq \mathbb{P}^{3}, X_{3} \cap X_{2}=F=X_{3} \cap X_{1}$. If $X$ contains $X_{4}$ then $X_{4}$ cuts a plane on some $X_{i}, i \leq 3$, hence $X_{4}$ intersects $E_{1}$ or passes through $E_{2}$, but this is not possible by Lemma 4 . We have $i i$ ).

2d) $X_{3} \simeq \mathbb{P}^{3}, X_{3} \cap X_{1}$ is a plane $F^{\prime}, X_{3} \cap X_{2}$ is a line $L \subset F^{\prime}$. Unless $L=E_{1}$ we get $F^{\prime}=F$, the previous considered case. If $X$ contains $X_{4}$ then $X_{4}$ cuts a plane on some $X_{i}, i \leq 3$. If $X_{4}$ cuts a plane on $X_{1}$, or on $X_{3}$, it intersects $E_{1}$ at least at a point $P$, but this is not possible by Lemma 4 . If $X_{4}$ cuts a plane $F^{\prime \prime}$ on $X_{2}$, then it must also cut a line $L^{\prime} \subset F^{\prime \prime}$ on $X_{1}$ hence $F^{\prime \prime}=F$ and $X_{4}$ intersects $E_{1}$ in this case too. We have $i i i)$.

Case 3: $X_{2} \simeq \mathbb{P}^{3}$, intersecting $X_{1}$ along a plane $F$. $X$ cannot be $X_{1} \cup X_{2}$ because $N \geq 7$. Let us consider $X_{3}$.

$3 a) X_{3}$ is a quadric cone. By Lemma $8, X_{3} \cap X_{1}$ is a plane $F^{\prime}$. If $F^{\prime}=F$ we can argue as in Case 2c) changing the role of $X_{2}$ and $X_{3}$. If $F^{\prime} \neq F, X_{3} \cap X_{2}=E_{1}$ because $F^{\prime} \cap X_{2}=E_{1}$ (recall Corollary $1 \mathrm{ii}$ )), moreover the vertex $E_{3}$ of $X_{3}$ cannot be a line, otherwise $E_{3} \cap E_{1}$ would be non empty and this is not possible by Lemma 4. Hence $E_{3}$ is a point on $F^{\prime}, E_{3} \notin E_{1}$. We can argue as in Case $2 d$ ) changing the role of $X_{2}$ and $X_{3}$.

3b) $X_{3} \simeq \mathbb{P}^{3}, X_{3} \cap X_{1}$ is a plane $F^{\prime} \neq F, X_{3} \cap X_{2}$ is a line $L \subset F^{\prime}$. Necessarily $L=E_{1}$ (otherwise $F^{\prime}=F$ ). If $X$ contains $X_{4}$, then $X_{4}$ cuts a plane on some $X_{i}$, $i \leq 3$. In any case $X_{4}$ intersects $E_{1}$ at least at a point $P$, but this is not possible by Lemma 4. Moreover $X$ cannot be $X_{1} \cup X_{2} \cup X_{3}$ as $N \geq 7$, so we have no possibilities.

3c) $X_{3} \simeq \mathbb{P}^{3}, X_{3} \cap X_{1}=F=X_{3} \cap X_{2}$. We can argue as in the previous case and we have no possibilities.

3d) $X_{3} \simeq \mathbb{P}^{3}, X_{3} \cap X_{2}$ is a plane $\Pi \neq F, X_{3} \cap X_{1}$ is a line $L \subset \Pi$. If $L=E_{1}$ $X$ cannot contain $X_{4}$ because $X_{4}$ would intersect $E_{1}$ in any case and this is not possible by Lemma 4; on the other hand $X$ cannot be $X_{1} \cup X_{2} \cup X_{3}$ as $N \geq 7$, so we can assume that $L \neq E_{1}$. Let $H$ be $L \cap E_{1}$, note that no other component can pass through $H$ by Lemma 4 . Let us consider $X_{4}$. If $X_{4}$ is a quadric cone then it must cut a plane on $X_{1}$ by Lemma 8 , hence it would pass through $H$ : contradiction. Therefore $X_{4} \simeq \mathbb{P}^{3}$. $X_{4}$ cuts a plane on some $X_{i}$ with $i \leq 3$, but $X_{4} \cap X_{1}$ cannot be a plane, otherwise $X_{4}$ would contain $H$. If $X_{4} \cap X_{3}$ is a plane then $X_{4} \cap X_{1}$ would be a line, necessarily contained in this plane (recall Corollary 1 ii)), but, as $X_{3} \cap X_{1}=L$, it would imply: $X_{4} \cap X_{1}=L$, hence $H \in X_{4}$ : contradiction. Hence there is only one possibility: $X_{4} \cap X_{2}$ is a plane $\Pi_{4}$ (not passing through $H$ ), $X_{4} \cap X_{1}$ is a line $L_{4} \subset F, X_{4} \cap X_{3}$ is the line $\Pi_{4} \cap \Pi$.

Now let us proceed by induction on the number of components of $X$. Let us put $L_{3}=L$ and $\Pi_{3}=\Pi$. In this subcase Proposition 6 is true when $X$ has $r=4$ components. Let us prove Proposition 6 assuming that it is true when $X$ has $4,5, \ldots, r-1$ components and considering the case in which $X$ has $r \geq 5$ components. Let us consider $X_{r}$. If $X_{r}$ is a quadric cone then it must cut a plane on $X_{1}$ by Lemma 8, hence it would pass through $H$ : contradiction. Therefore $X_{r} \simeq \mathbb{P}^{3}$. $X_{r}$ cuts a plane on some $X_{i}$ with $i \leq r-1$, but $X_{r} \cap X_{1}$ cannot be a plane, otherwise $X_{r}$ would contain $H$. If $X_{r} \cap X_{i}$ is a plane for some $i=3, \ldots, r-1$, then $X_{r} \cap X_{1}$ 
would be a line, necessarily contained in this plane (recall Corollary $1 \mathrm{ii}$ )), but, as $X_{i} \cap X_{1}=L_{i} \subset F=X_{1} \cap X_{2}$ by induction, this would imply: $X_{r} \cap X_{1}=L_{i}$, hence there would be at least a point in $X_{1} \cap X_{2} \cap X_{i} \cap X_{r}$ and this is not possible by Lemma 4. Hence there is only one possibility: $X_{r} \cap X_{2}$ is a plane $\Pi_{r}$ (in generic position in $\left.X_{2}\right), X_{r} \cap X_{1}$ is a line $L_{r} \subset F, X_{r} \cap X_{i}$ is the line $\Pi_{r} \cap \Pi_{i}$ for $i=3, \ldots, r-1$, and we have $i v$ ).

Proposition 7. Let $X \subset \mathbb{P}^{N}$ be a 3-projectable Veronese variety, $\operatorname{dim}(X)=3$. Let us assume that $X$ does not contain cones of degree 3 , or of degree 2 having a line as vertex, and that $X$ contains a cone $X_{i}$ of degree 2 having a point $E_{i}$ as vertex. Then:

i) $X=X_{1} \cup X_{2} \cup \ldots \cup X_{r} \subset \mathbb{P}^{r+3}, r \geq 4$; $X_{1}$ is a quadric cone having a point $E_{1}$ as vertex, $X_{i} \simeq \mathbb{P}^{3}$ for $i \geq 2 ; X_{1} \cap X_{2}$ is a plane $F ; X_{i} \cap X_{2}$ are planes in generic position in $X_{2}$ intersecting lines $L_{i} \subset F$ in generic position for $i \geq 3$; possibly one of the components $X_{p}, 3 \leq p \leq r$, is exceptional: it intersects $X_{1}$ along another plane $F^{\prime}$, cutting $F$ along a line $l$ in generic position with respect to the set $\left\{L_{i}, i \neq p\right\}$

ii) $X=X_{1} \cup X_{2} \cup \ldots \cup X_{r} \subset \mathbb{P}^{r+3}, r \geq 4 ; X_{1}$ is a quadric cone having a point $E_{1}$ as vertex, $X_{i} \simeq \mathbb{P}^{3}$ for $i \geq 2 ; X_{1} \cap X_{2}$ is a plane $F ; X_{i} \cap X_{2}$ are planes in generic position in $X_{2}$ intersecting lines $L_{i} \subset F$ in generic position for $i=3, \ldots, r-1$; there exists a fixed $j, 3 \leq j \leq r-1$, such that $X_{r} \cap X_{j}$ is a plane and $X_{r} \cap X_{1}=L_{j}$ $\left(E_{1} \notin L_{j}\right)$;

iii) $X=X_{1} \cup X_{2} \cup X_{3} \cup X_{4} \subset \mathbb{P}^{7} ; X_{1}$ is a quadric cone having a point $E_{1}$ as vertex, $X_{i} \simeq \mathbb{P}^{3}$ for $i \geq 2 ; X_{1} \cap X_{2} \cap X_{3}$ is a plane $F ; X_{4} \cap X_{2}$ is a plane $\Pi$, $X_{4} \cap X_{3}=X_{4} \cap X_{1}$ is a line $L=\Pi \cap F$, not passing through $E_{1}$, or $X_{4} \cap X_{3}$ is a plane $\Pi, X_{4} \cap X_{2}=X_{4} \cap X_{1}$ is a line $L=\Pi \cap F$, not passing through $E_{1}$.

Proof. As usual we can assume that $X_{1}$ is a quadric cone having a point $E_{1}$ as vertex by Proposition 1. By the previous Propositions we know that all other components of $X$ are linear spaces. $X_{2} \cap X_{1}$ is a plane $F$. We consider two cases.

Case 1: for any $i \geq 3, X_{i} \cap X_{1}$ is a line $L_{i}$. Let us consider $X_{3}$. $X_{3}$ must cut a plane $\Pi_{3}$ on $X_{2}$ and $L_{3}=\Pi_{3} \cap F$ on $X_{1} . X$ must contain another component as $N \geq 7$.

Let us consider $X_{4}$. $X_{4}$ has to cut a plane on $X_{3}$ or on $X_{2}$ (or on both). If $X_{4}$ cuts a plane on $X_{3}$ containing the line $X_{4} \cap X_{1}=L_{4}$ then $L_{4}=L_{3}$ as $X_{3} \cap X_{1}=L_{3}$. This case is possible only if $E_{1} \notin L_{3}$, otherwise we would get a contradiction with Lemma 4 , and in this case no other component of $X$ can intersect $L_{4}=L_{3} \subset F$ by Corollary 4 , on the other hand another component $X_{5}$ would cut a plane on some $X_{i}$ with $2 \leq i \leq 4$, hence $X_{5}$ would intersect $L_{3}$ : contradiction. Then we get $i i$ ) with $r=4$ and $j=3$.

If $X_{4}$ cuts a plane $\Pi_{4}$ on $X_{2}$, then $X_{4} \cap X_{1}=\Pi_{4} \cap F$ is a line $L_{4}$, while $X_{4} \cap X_{3}$ is the line $\Pi_{4} \cap \Pi_{3}$ (the position of $E_{1}$ with respect to $L_{4}$ is not important). Here we get $i$ ) with $r=4$.

Now we can proceed by induction on the number of components of $X$. Let us assume that if $X$ has $r-1$ components, $r \geq 5$, such that for any $i \geq 3, X_{i} \cap X_{1}$ is a line $L_{i}$ and let us assume that $i$ ) or $i i$ ) holds for $X$. Let us prove that, if $X$ has $r$ components, $r \geq 5$, such that for any $i \geq 3, X_{i} \cap X_{1}$ is a line $L_{i}$, then $i$ ) or $i i$ ) holds for $X$. Let us consider $Y:=X_{1} \cup \ldots \cup X_{r-1}$; by induction we know that $i$ ) or $i$ i) holds for $Y$. In fact $i i$ ) cannot hold: $X_{r-1} \cap X_{1}=L_{r-1}=L_{j} \subset F$ for some $j$ with $3 \leq j \leq r-2 ; X_{r}$ must cut a plane on some $X_{i}$ with $2 \leq i \leq r-1$ and a line 
$L_{r}$ on $X_{1}$ such that $L_{r}$ is contained in that plane, hence $L_{r} \subset F$ and $L_{r}$ intersects $L_{r-1}=L_{j}$, then there would be at least a point on five components of $X: X_{1}, X_{2}$, $X_{j}, X_{r-1}, X_{r}$ : contradiction with Corollary 4. Therefore $i$ ) holds for $Y$. Now $X_{r}$ must cut a plane on some $X_{i}$ with $2 \leq i \leq r-1$ and a line $L_{r}$ on $X_{1}$ such that $L_{r}$ is contained in that plane, hence $L_{r} \subset F$. If $L_{r}$ is distinct from $L_{j}$ with $3 \leq j \leq r-1$, and necessarily in general position on $F$ to avoid contradiction with Corollary 4 , then we have $i$ ) for $X$; if $L_{r}$ concides with a line $L_{j}$ with $3 \leq j \leq r-1$, necessarily not passing through $E_{1}$, then we have $i i$ ) for $X$.

Case 2: there exists at least a component $X_{i}, i \geq 3$, such that $X_{i} \cap X_{1}$ is a plane.

2a) $X_{3} \cap X_{1}$ is a plane. Firstly let us assume that $X_{3} \cap X_{1}:=\Pi \neq F$ and $\Pi \cap F=\emptyset$ (then $X_{3} \cap X_{2}=E_{1}$, recall Corollary $\left.1 i i\right)$ ). $X$ must contain another component as $N \geq 7$. Let us consider $X_{4}$. $X_{4}$ has to cut a plane on $X_{i}, i \leq 3$. If $X_{4} \cap X_{1}$ is a plane $X_{4}$ contains $E_{1}$; if $X_{4} \cap X_{2}$ is a plane then $X_{4}$ cuts a line or a point on $X_{3}$, hence $E_{1} \in X_{4}$; if $X_{4} \cap X_{3}$ is a plane then $X_{4}$ cuts a line or a point on $X_{2}$, hence $E_{1} \in X_{4}$; therefore $X_{4}$ contains $E_{1}$ in any case, but this is not possible by Lemma 4 .

Secondly let us assume that $X_{3} \cap X_{1}=F$. $X$ must contain another component $X_{4}$, as $N \geq 7$, and, by arguing as above, we get $X_{4} \cap X_{2}$ is a plane $\Pi$ intersecting $F$ (and $X_{1}$ and $X_{3}$ ) along a line $L$ not passing through $E_{1}$, or $X_{4} \cap X_{3}$ is a plane $\Pi$ intersecting $F$ (and $X_{1}$ and $X_{2}$ ) along a line $L$ not passing through $E_{1}$. In both cases $X$ cannot contain another component $X_{5}: X_{5}$ would cut a plane on $X_{i}$, $i \leq 4$, hence it would cut $L$ : contradiction with Corollary 4 , or it would contain $E_{1}$ : contradiction with Lemma 4 . We get $\left.i i i\right)$.

Thirdly let us assume that $X_{3} \cap X_{1}:=\Pi \neq F$ and $\Pi \cap F:=l$ is a line. $X$ must contain another component $X_{4}$, as $N \geq 7$, and $X_{4}$ cuts a plane on $X_{i}, i \leq 3$. As $X_{4} \cap X_{1}$ cannot be a plane, by looking at $\mathbb{T}_{E_{1}}$, we have that $X_{4} \cap X_{3}$ or $X_{4} \cap X_{2}$ is a plane. We can assume that $X_{4} \cap X_{2}:=\Pi_{4}$ is a plane intersecting $F$ along a line $L_{4}$, cutting $l$ at a point $P_{4} \neq E_{1}$. Note that $X_{3} \cap X_{4}=P_{4} . X$ can contain another component $X_{5}$. It cannot cut neither a plane on $X_{1}$ nor on $X_{3}$ or $X_{4}$ : if $X_{5} \cap X_{4}$ would be a plane it would pass through $P_{4}$ (recall Corollary $1 \mathrm{ii}$ )) and this is not possible by Corollary 4. Hence $X_{5} \cap X_{2}$ is a plane $\Pi_{5}$ cutting $F$ along a line $L_{5}$ in generic position with respect to $l$ and $L_{4}$ and $i$ ) holds for $X_{1} \cup X_{2} \cup X_{4} \cup X_{5}$. Now we can proceed as in Case 1 because, by arguing as above, the other components $X_{i}$ must cut $X_{2}$ along a plane $\Pi_{i}$, cutting $F$ along a line $L_{i}$ in generic position with respect to the previous ones. Note that $X_{i}$ cannot cut $F$ along a line $L_{j}, i<r$, (as in $i i)$ ) otherwise at least five components of $X$ would pass through $l \cap L_{j}$. We get i) with $p=3$.

2b) $X_{3} \cap X_{1}$ is a line. By arguing as in Case 1 we get $X_{3} \cap X_{2}$ is a plane $\Pi_{2}$, intersecting $F$ and $X_{1}$ along a line $L_{3} . X$ must contain another component as $N \geq 7$. Let us consider $X_{4}$. $X_{4}$ has to cut a plane on some $X_{i}, i \leq 3$.

Let us assume that $X_{4}$ cuts a plane $F^{\prime}$ on $X_{1}$. If $F^{\prime} \neq F$ and $F^{\prime} \cap F=E_{1}$, then $X_{4} \cap X_{3}=\emptyset$ and this is not possible by Lemma $\left.2 i i\right)$. If $F^{\prime}=F$ we have $X_{4} \cap X_{3}=L_{3}$. $X$ cannot have another component $X_{5}: X_{5}$ would cut a plane on $X_{i}, i \leq 4$, hence it would cut $L_{3}$ : contradiction with Corollary 4 or it would contain $E_{1}$ : contradiction with Lemma 4 . We get $\left.i i i\right)$. If $F^{\prime} \neq F$ and $F^{\prime} \cap F:=l$ is a line, we are in Case $2 a$ ) with $X_{4}$ in the role of $X_{3}$. We can proceed as in $2 a$ ) and we get $i)$, with $p=4$. 
Let us assume that $X_{4}$ cuts a line $L_{4}$ on $X_{1}$, then $Y:=X_{1} \cup X_{2} \cup X_{3} \cup X_{4}$ is as in Case 1 and we get $i i)$ or $i$ ) for $Y$. Only in this second case $\left(X_{4} \cap X_{2}\right.$ is a plane $\Pi_{2}$ cutting a line $L_{4}$ on $F$ in generic position with respect $L_{3}$ and $E_{1}$ ), there can be another component $X_{5}$ for $X . X_{5}$ has to cut a plane on some $X_{i}, i \leq 4$. By arguing as above we can exclude that $X_{5} \cap X_{1}$ is a plane different from $F$. On the other hand $X_{5} \cap X_{1}$ cannot be $F$, otherwise there would be at least a point on five components of $X$ (i.e. $L_{4} \cap L_{3} \subset F$ ) and this would be a contradiction with Corollary 4. Therefore $X_{5} \cap X_{1}$ must be a plane intersecting $F$ along a line $l$ and we get $i$ ) with $p=5$, or $X_{5} \cap X_{1}$ is a line and so on. By repeating the above arguments it is easy to see that we get $i$ ) with an exceptional component $X_{p}$.

\section{Proof of Theorem 2}

In this section we give the proof of Theorem 2 by showing that only the nine varieties listed in Theorem 2 are 3-projectable among all remaining varieties, after having imposed necessary conditions in the previous sections. In any case it suffices to prove or disprove that $\operatorname{dim}\left(\bigcup_{P \in X} T_{P}(X)\right) \leq m+3$.

Firstly let us consider the case in which all components of $X$ are linear spaces. By Lemma 6 we know that the only possibilities for $X$ are $i$ ) and $i i$ ) described in that Lemma, with $r \geq 5$ by Lemma $5 i$ ). Theorem 5 says that such an $X$ is in fact 3 -projectable. We get 1) and 2).

Let us assume $m \geq 4$. By Section 5 we know that the possibilities for $X$ are the three cases described in Theorem 7 .

In the first case $X \subset \mathbb{P}^{9}$. If we consider a point $P \in X_{1} \cap X_{2} \cap X_{3}$ we have, for any $i=1,2,3, T_{P}\left(X_{i}\right) \simeq \mathbb{P}^{4}$ is generated by $\left\langle E_{i} \cup P\right\rangle$ and the tangent plane at $\pi_{i}(P)$ to the smooth quadric $Q_{i}$, base of the cone $X_{i}$, where $\pi_{i}$ is the natural projection from $E_{i}$ onto $Q_{i}$. Let $L_{i}$ be the line $X_{1} \cap X_{2} \cap X_{3} \cap Q_{i}$, then $T_{P}(X) \simeq \mathbb{P}^{6}$ is generated by $X_{1} \cap X_{2} \cap X_{3}$ and the 3 lines $L_{P, i}^{\prime} \subset Q_{i}$ intersecting $L_{i}$ at $\pi_{i}(P)$. Viceversa, if we take 3 generic points $R_{i} \in Q_{i}$, if we consider the 3 points $R_{i}^{\prime}$ intersected on $L_{i}$ by the unique line $L_{R_{i}}^{\prime}$ of $Q_{i}$ passing through $R_{i}$, not belonging to the ruling of $L_{i}$, if we consider the point $P:=\left\langle E_{1} \cup R_{1}^{\prime}\right\rangle \cap\left\langle E_{2} \cup R_{2}^{\prime}\right\rangle \cap\left\langle E_{3} \cup R_{3}^{\prime}\right\rangle \in X_{1} \cap X_{2} \cap X_{3} \simeq \mathbb{P}^{3}$, then $T_{P}(X)=\left\langle\left(X_{1} \cap X_{2} \cap X_{3}\right) \cup L_{R_{1}}^{\prime} \cup L_{R_{2}}^{\prime} \cup L_{R_{3}}^{\prime}\right\rangle$. It follows that the union of all the 6-dimensional linear spaces $T_{P}(X)$, for generic points $P \in X_{1} \cap X_{2} \cap X_{3}$, has dimension 9 , and $X$ is not 3 -projectable into $\mathbb{P}^{7}$.

In the second case $X \subset \mathbb{P}^{8}$. We can argue as in the previous case and we can prove that the union of all the 6 -dimensional linear spaces $T_{P}(X)$, for generic points $P \in X_{1} \cap X_{2} \cap X_{3}$, has dimension 8 , and $X$ is not 3-projectable into $\mathbb{P}^{7}$.

In the third case $X \subset \mathbb{P}^{8}$. Let us consider the line $L:=X_{1} \cap X_{2} \cap X_{3} \cap X_{4}$ and recall that $L$ is disjoint from the line $E_{1}$, vertex of $X_{1}$ and that $X_{1} \cap\left(X_{2} \cup\right.$ $\left.X_{3} \cup X_{4}\right)=F:=X_{1} \cap X_{2} \simeq \mathbb{P}^{3}$. For any point $Q \in L, \operatorname{dim}\left[T_{Q}(X)\right]=7$ and $T_{Q}(X)=\left\langle T_{Q}\left(X_{1}\right) \cup X_{2} \cup X_{3} \cup X_{4}\right\rangle$. As the projection of $L$ from $E_{1}$ is a line on the 2-dimensional smooth quadric $B_{1}$ which is the base of $X_{1}$, when $Q$ moves in $L$, $T_{Q}\left(X_{1}\right)$ varies in a pencil of 4 -dimensional linear spaces, containing $F$, spanned by $E_{1}$ and the tangent plane to $B_{1}$ at $\pi_{1}(Q)$, hence $\operatorname{dim}\left(\bigcup_{Q \in L} T_{Q}(X)\right)=8$ and $X$ is not 3-projectable into $\mathbb{P}^{7}$.

From now on let us assume that $m=3$. 
By Proposition 4 we know that, if $X$ contains a scroll, the possibilities are those described in that Proposition.

In the first case, let $F$ be the fixed fibre of the scroll $X_{1}$ where all components of $X$ intersect. For any point $P \in F, T_{p}(X)=\left\langle T_{P}\left(X_{1}\right) \cup X_{2} \cup X_{3}\right\rangle \simeq \mathbb{P}^{5}$, moreover for any other point $P^{\prime} \in F, T_{P^{\prime}}(X)=\left\langle T_{P^{\prime}}\left(X_{1}\right) \cup X_{2} \cup X_{3}\right\rangle \neq T_{p}(X)$, because $T_{P}\left(X_{1}\right) \neq T_{P^{\prime}}\left(X_{1}\right)$ by Lemma 7 and $\left\langle X_{2} \cup X_{3}\right\rangle \cap\left\langle X_{1}\right\rangle=F$. It follows that the union of all the 5 -dimensional linear spaces $T_{P}(X)$, for generic points $P \in F$, has dimension 7 , and $X$ is not 3 -projectable into $\mathbb{P}^{6}$.

In the second case $X \subset \mathbb{P}^{8}$. We can argue as in the previous case and we can prove that the union of all the 6 -dimensional linear spaces $T_{P}(X)$, for generic points $P \in F$, has dimension 8 , and $X$ is not 3 -projectable into $\mathbb{P}^{6}$.

Proposition 5 describes the unique possibility for $X$ when it contains a cubic cone. $X \subset \mathbb{P}^{7}$, to prove that $X$ is 3 -projectable into $\mathbb{P}^{6}$ it suffices to show that $\operatorname{dim}\left(\bigcup_{P \in X} T_{P}(X)\right) \leq 6$. Obviously it suffices to control points belonging to $X_{1} \cap X_{2} \simeq$ $\mathbb{P}^{2}$, because if $P$ belongs to a unique $X_{i}, T_{P}(X) \subset\left\langle X_{i}\right\rangle \subset \mathbb{P}^{7}$.

If $P \in\left(X_{1} \cap X_{2}\right) \backslash\left(E_{1} \cup E_{2}\right), T_{p}(X)=\left\langle T_{P}\left(X_{1}\right) \cup T_{P}\left(X_{2}\right)\right\rangle \simeq \mathbb{P}^{4}$

so that $\operatorname{dim}\left(\bigcup_{P \in\left(X_{1} \cap X_{2}\right) \backslash\left(E_{1} \cup E_{2}\right)} T_{P}(X)\right) \leq 6$.

If $P \in E_{1}, T_{p}(X)=\left\langle T_{P}\left(X_{1}\right) \cup T_{P}\left(X_{2}\right)\right\rangle \simeq \mathbb{P}^{6}$ and this linear space is fixed as $P$ varies in $E_{1}$. If $P=E_{2}, T_{p}(X)=\left\langle T_{P}\left(X_{1}\right) \cup T_{P}\left(X_{2}\right)\right\rangle \simeq \mathbb{P}^{5}$. Hence $X$ is 3 projectable into $\mathbb{P}^{6}$ and we get 3$)$.

By Proposition 6 we know that, if $X$ does not contain cubic cones but it contains a quadric cone having a line as vertex, the possibilities are those described in that Proposition. Let us examine them.

i) $X \subset \mathbb{P}^{8}$. For any generic $P \in F, T_{p}(X)=\left\langle T_{P}\left(X_{1}\right) \cup T_{P}\left(X_{2}\right) \cup T_{P}\left(X_{3}\right)\right\rangle \simeq$ $\mathbb{P}^{5}$. As $P$ varies in $F, T_{P}\left(X_{1}\right)$ is fixed, but $T_{P}\left(X_{2}\right)$ and $T_{P}\left(X_{3}\right)$ are generated, respectively, by $F$ and the two lines, of the smooth base quadrics $Q_{2}$ and $Q_{3}$, different from $L_{2}:=F \cap Q_{2}, L_{3}:=F \cap Q_{3}$ and passing through the points $\pi_{2}(P)$ and $\pi_{3}(P)$, where $\pi_{i}$ is the natural projection from the vertex $E_{i}$ of the cone $X_{i}$ onto its base $Q_{i}$. Viceversa, if we take two generic points $R_{2} \in Q_{2}$ and $R_{3} \in Q_{3}$, if we consider the two points $R_{i}^{\prime}$ intersected on $L_{i}$ by the unique line $L_{R_{i}}^{\prime}$ of $Q_{i}$ passing through $R_{i}$, not belonging to the ruling of $L_{i}(i=1,2)$, if we consider the point $P:=\left\langle E_{2} \cup R_{2}^{\prime}\right\rangle \cap\left\langle E_{3} \cup R_{3}^{\prime}\right\rangle \in F \simeq \mathbb{P}^{2}$, then $T_{P}(X)=\left\langle T_{P}\left(X_{1}\right) \cup L_{R_{2}}^{\prime} \cup L_{R_{3}}^{\prime}\right\rangle$. We claim that $\operatorname{dim}\left[\bigcup_{\text {generic }}{ }_{P \in F} T_{P}(X)\right]=7$, excluding the $x$-projectability of $X$. Indeed, since the intersection of two general elements of this family is not $\mathbb{P}^{5}$, generic $P \in F$ $T_{P}(X)$ cannot be contained in a linear space of dimension 6 . Moreover, no non-linear 6-dimensional variety contains a 2-dimensional family of linear spaces of dimension 5 (cut with a general linear subspace and use that a plane is the unique integral surface of a projective space containing a 2-dimensional family of lines). It follows that the union of all the 5 -dimensional linear spaces $T_{P}(X)$, for generic points $P \in F$, has dimension 7 . Hence $X$ is not 3 -projectable into $\mathbb{P}^{6}$.

ii) $X \subset \mathbb{P}^{7}$. To prove that $X$ is 3 -projectable into $\mathbb{P}^{6}$ it suffices to show that $\operatorname{dim}\left(\bigcup_{P \in X} T_{P}(X)\right) \leq 6$. Obviously it suffices to control points belonging to two or more components of $X$, because if $P$ belongs to a unique $X_{i}, T_{P}(X) \subset\left\langle X_{i}\right\rangle \subset \mathbb{P}^{7}$.

If $P \in F \backslash\left(E_{1} \cup E_{2}\right), T_{p}(X)=\left\langle T_{P}\left(X_{1}\right) \cup T_{P}\left(X_{2}\right) \cup T_{P}\left(X_{3}\right)\right\rangle \simeq \mathbb{P}^{5}$. As $P$ varies in $F \backslash\left(E_{1} \cup E_{2}\right), T_{P}\left(X_{1}\right)$ and $T_{P}\left(X_{3}\right)$ are fixed, while $T_{P}\left(X_{2}\right)$ is generated by 
$F$ and the lines, of the smooth base quadrics $Q_{2}$, different from $F \cap Q_{2}$, and passing through the points $\pi_{2}(P)$. Hence the union of all the 5 dimensional linear spaces $T_{P}(X)$, for points $P \in F \backslash\left(E_{1} \cup E_{2}\right)$, has dimension 6. If $P \in E_{1}$, $T_{p}(X)=\left\langle T_{P}\left(X_{1}\right) \cup T_{P}\left(X_{2}\right) \cup T_{P}\left(X_{3}\right)\right\rangle \simeq \mathbb{P}^{6}$ and this space is fixed as $P$ varies in $E_{1}$. If $P=E_{2}, \operatorname{dim}\left[T_{p}(X)\right]=6$. Hence $X$ is 3 -projectable into $\mathbb{P}^{6}$ and we get 4$)$.

iii) $X \subset \mathbb{P}^{7}$. To prove that $X$ is 3 -projectable into $\mathbb{P}^{6}$ it suffices to show that $\operatorname{dim}\left(\bigcup_{P \in X} T_{P}(X)\right) \leq 6$. Obviously it suffices to control points belonging to two or more components of $X$.

If $P \in F \backslash\left(E_{1} \cup E_{2}\right), T_{P}(X)=\left\langle T_{P}\left(X_{1}\right) \cup T_{P}\left(X_{2}\right)\right\rangle \simeq \mathbb{P}^{4}$,

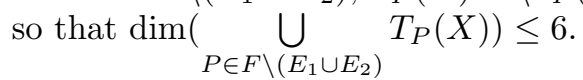

If $P \in F^{\prime} \backslash\left(E_{1}\right), T_{P}(X)=\left\langle T_{P}\left(X_{1}\right) \cup T_{P}\left(X_{3}\right)\right\rangle \simeq \mathbb{P}^{4}$, so that $\operatorname{dim}\left(\bigcup_{P \in F^{\prime} \backslash\left(E_{1}\right)} T_{P}(X)\right) \leq 6$.

If $P \in E_{1}, T_{p}(X)=\left\langle T_{P}\left(X_{1}\right) \cup T_{P}\left(X_{2}\right) \cup T_{P}\left(X_{3}\right)\right\rangle \simeq \mathbb{P}^{6}$ and this space is fixed as $P$ varies in $E_{1}$. If $P=E_{2}, \operatorname{dim}\left[T_{p}(X)\right]=5$. Hence $X$ is 3 -projectable into $\mathbb{P}^{6}$ and we get 5$)$.

iv) $X \subset \mathbb{P}^{r+3}$. To prove that $X$ is 3 -projectable into $\mathbb{P}^{6}$ it suffices to show that $\operatorname{dim}\left(\bigcup_{P \in X} T_{P}(X)\right) \leq 6$. Obviously it suffices to control points belonging to two or more components of $X$.

If $P \in F \backslash\left(E_{1} \cup L_{2} \cup \ldots \cup L_{r}\right), T_{p}(X)=\left\langle T_{P}\left(X_{1}\right) \cup T_{P}\left(X_{2}\right)\right\rangle \simeq \mathbb{P}^{4}$, so that $\operatorname{dim}\left(\cup T_{P}(X)\right) \leq 6$.

$P \in F \backslash\left(E_{1} \cup L_{2} \cup \ldots \cup L_{r}\right)$

If $P \in E_{1} \backslash\left[\left(E_{1} \cap L_{2}\right) \cup \ldots \cup\left(E_{1} \cap L_{r}\right)\right], T_{p}(X)=\left\langle T_{P}\left(X_{1}\right) \cup T_{P}\left(X_{2}\right)\right\rangle \simeq \mathbb{P}^{5}$ and this space is fixed as $P$ varies in $E_{1} \backslash\left[\left(E_{1} \cap L_{2}\right) \cup \ldots \cup\left(E_{1} \cap L_{r}\right)\right]$.

If $P \in L_{i} \backslash\left[\left(E_{1} \cap L_{i}\right) \underset{j \geq 3, j \neq i}{\bigcup}\left(L_{i} \cap L_{j}\right)\right], i \geq 3, T_{p}(X)=\left\langle T_{P}\left(X_{1}\right) \cup T_{P}\left(X_{2}\right) \cup T_{P}\left(X_{i}\right)\right\rangle$ $\simeq \mathbb{P}^{5}$ and this space is fixed as $P$ varies in $L_{i} \backslash\left[\left(E_{1} \cap L_{i}\right) \underset{j \geq 3, j \neq i}{\bigcup}\left(L_{i} \cap L_{j}\right)\right]$.

If $P \in\left(X_{i} \cap X_{j}\right) \backslash\left(F \underset{k \geq 3,}{\bigcup_{k \neq i, k \neq j}} X_{k}\right), i, j \geq 3, T_{p}(X)=\left\langle T_{P}\left(X_{i}\right) \cup T_{P}\left(X_{j}\right) \cup T_{P}\left(X_{2}\right)\right\rangle$ $\simeq \mathbb{P}^{5}$ and this space is fixed as $P$ varies in $\left(X_{i} \cap X_{j}\right) \backslash\left(F \underset{k \geq 3, k \neq i, k \neq j}{\bigcup_{k}} X_{k}\right.$.

If $P$ is in the remaining discrete sets of a finite number of points we know that $\operatorname{dim}\left[T_{p}(X)\right] \leq 6$ because this necessary condition, given by Corollary 4 , was checked in the proof of Proposition 6 . Hence $X$ is 3 -projectable into $\mathbb{P}^{6}$ and we get 6 ).

By Proposition 7 we know that the last three possibilities for $X$ are those described in that Proposition. Let us examine them.

i) We can argue as in the previous case $i v$ ) of Proposition 6 and conclude that $X$ is 3 -projectable into $\mathbb{P}^{6}$, it is 7 ). Note that the existence of an exceptional component $X_{p}$ is not important, since for any generic point $P \in l:=X_{p} \cap F$ we have $T_{p}(X)=\left\langle T_{P}\left(X_{1}\right) \cup T_{P}\left(X_{2}\right) \cup T_{P}\left(X_{p}\right)\right\rangle \simeq \mathbb{P}^{5}$ and these linear spaces are fixed for generic $P \in l$. For the remaining discrete sets of points on $l$ we know that $\operatorname{dim}\left[T_{p}(X)\right] \leq 6$.

ii) We can argue as in the previous case $i v)$ of Proposition 6 unless $P \in X_{r}$.

If $P \in\left(X_{r} \cap X_{j}\right) \backslash L_{j}, T_{p}(X)=\left\langle T_{P}\left(X_{j}\right) \cup T_{P}\left(X_{r}\right)\right\rangle \simeq \mathbb{P}^{4}$ 
and this space is fixed as $P$ varies in $\left(X_{r} \cap X_{j}\right) \backslash L_{j}$.

If $P \in L_{j} \backslash\left(\bigcup_{k \geq 3, k \neq r, k \neq j} X_{k}\right), T_{p}(X)=\left\langle T_{P}\left(X_{1}\right) \cup T_{P}\left(X_{2}\right) \cup T_{P}\left(X_{j}\right) \cup T_{P}\left(X_{r}\right)\right\rangle \simeq$ $\mathbb{P}^{6}$ and this space is fixed as $P$ varies in $L_{j} \backslash\left(\bigcup_{k \geq 3, k \neq r, k \neq j} X_{k}\right)$.

If $P$ is in the remaining discrete sets of a finite number of points we know that $\operatorname{dim}\left[T_{p}(X)\right] \leq 6$ because this necessary condition, given by Corollary 4 , was checked in the proof of Proposition 7. Hence $X$ is 3 -projectable into $\mathbb{P}^{6}$ and we get 8 ).

iii) We can argue as in previous cases: for $P \in F \backslash L, P \in \Pi \backslash L, P \in L$ the tangent space is fixed as $P$ varies in these sets and $\operatorname{dim}\left[T_{P}(X)\right] \leq 6$. If $P=E_{1}$ $\operatorname{dim}\left[T_{p}(X)\right]=5$. Hence $X$ is 3 -projectable into $\mathbb{P}^{6}$ and we get 9 ).

As there are no other possibilities for $X$ we have proved Theorem 2 .

\section{REFERENCES}

[A1] B.Adlandsvik: "Joins and higher secant varieties" Math. Scand. 62 (1987), p. 213-222.

[A2] B.Adlandsvik: "Varieties with an extremal numbers of degenerate higher secant varieties" J. Reine Angew. Math. 392 (1988) p. 16-26.

[A-B] A.Alzati-E.Ballico: "Reducible Veronese surfaces". To appear on Advances in Geometry.

[B-S] M.C.Beltrametti-A.J.Sommese: "The adjunction theory of complex projective varieties" Walter de Gruyter, Berlin, 1995.

[B-H] W.Bruns-J.Herzog: "Cohen-Macaulay rings" Cambridge studies in advanced math. 39, Cambridge Univ. Press 1993.

[C] M.L.Catalano-Johnson: "The Possible Dimensions of the Higher Secant Varieties" Amer. J. Math. 118 (2) (1996), p. 355-361.

[E] D.Eisenbud: "Commutative Algebra with a View Towards Algebraic Geometry" G.T.M. 150, Springer, New York, 1995.

[F] G.Fløystad: "Monads on projective spaces" Comm. Algebra 28 (12) (2000), p. 55035516.

[E-G-H-P] D.Eisenbud-M.Green-K.Hulek-S.Popescu: "Small schemes and varieties of minimal degree" Amer. J. Math. 128 (6) (2006), p. 1363-1389.

[J] K.W.Johnson: "Immersion and embeddings of projective varieties" Acta Math. 140 (1978), p. 49-74.

[Ha] R.Hartshorne: "Complete intersections and connectedness" Amer. J. Math. 84 (1962), p. 497-508.

[Ho] A.Holme: "Embedding obstructions for singular algebraic varieties in $\mathbb{P}^{N}$ " Acta Math. 135 (1975), p. 155-185.

[X] S.Xambó: "On projective varieties of minimal degree" Collectanea Math. 32 (2) (1981), p. $149-163$.

Dipartimento di Matematica Univ. Di Milano, via C. Saldini 50 20133-Milano (Italy)

E-mail address: alberto.alzati@unimi.it

Dipartimento di Matematica Univ. di Trento, via Sommarive 14 38050-Povo (TN) (ITALY)

E-mail address: ballico@science.unitn.it 